\title{
Vibration Exercise: The Potential Benefits
}

\author{
Author \\ D. J. Cochrane \\ Affiliation \\ Massey University, School of Sport \& Exercise, Palmerston North, New Zealand
}

Key words
tendon reflex
EMG
- heart rate
blood pressure
power
- force

accepted after revision

October 06, 2010

Bibliography

DOI http://dx.doi.org/

10.1055/s-0030-1268010

Published online:

December 16, 2010

Int J Sports Med 2011; 32:

75-99 ๑ Georg Thieme

Verlag KG Stuttgart · New York

ISSN 0172-4622

Correspondence

Dr. D. J. Cochrane

Massey University

School of Sport \& Exercise

Tennent Drive

4442 Palmerston North

New Zealand

Tel.: + 64/6/350 5799

Fax: $+64 / 6 / 3505661$

d.cochrane@massey.ac.nz

\section{Abstract \\ $\nabla$}

The aim of this review was to examine the physiological effects of vibration exercise (VbX), including the cardiovascular indices and to elucidate its potential use for those with compromised health. VbX has long been acknowledged as a potential modality in sport, exercise, and health sectors. Muscle force and power have been shown to increase after $\mathrm{VbX}$ for athletes, the aged and those with diseases, where neural factors are thought to be the main contributor. Further, similarities to the tonic vibration reflex have been used to propose that the muscle spindle plays a role in activating the muscle which could benefit those with compromised health. There is strong evidence that acute $\mathrm{VbX}$ can enhance upper and lower-body muscle power, and there is some indication that longer-term VbX can augment muscle power of upper and lower body extremities, although this is less convincing. It is not conclusive whether $\mathrm{VbX}$

\section{Introduction \\ $\nabla$}

Vibration exercise (VbX) is currently enjoying popularity as an alternative exercise modality for enhancing muscle activity, force and power $[1,12,60,105,108]$ while others have found little or no effect $[36,40,41]$. VbX has been suggested as an attractive and efficient complement to traditional forms of exercise for athletes, the aged and health compromised individuals. The muscle performance benefit supposedly occurs via neurogenic potentiation involving spinal reflexes and muscle activation, which is based on the tonic vibration reflex $[21,103]$. It has been purported that neural factors are responsible for the increases in muscle function, which are similar to those neural changes seen after several weeks of conventional resistance and power training increases force attributes. This has been fraught by the type and parameters used for various muscle contractions, and the different sample populations that have varied in chronological age, experience and training status. VbX provides an insufficient stimulus to enhance cardiovascular indices, where $\mathrm{VbX}$ cannot increase heart rate to the same extent as conventional aerobic exercise. But when conventional aerobic exercise is not possible, for example, in aged, cardiovascular compromised persons, VbX could be implemented at an early stage because it could provide a safe induction of a slight elevation of cardiovascular function indices while providing neural and myogenic benefits. In conclusion, $\mathrm{VbX}$ is a safe modality to increase physiological responses of reflex and muscle activity, and muscle function, for athletes, the aged and compromised health. However, further research should focus on the optimum dose relationship of frequency, amplitude and duration for the various populations.

$[13,14]$. Further, it has been purported that VbX augments muscle spindle activity that causes a stretch-reflex response and elicits a rapid but small change in muscle length [21], which is of importance to those with diseased tissue or neurological problems. The change in muscle activity is likely to evoke a small increase in oxygen uptake, which suggests muscle energy turnover exists where VbX may have a beneficial effect for cardiovascular indices, such as blood pressure, blood flow and heart rate.

Despite its wide use in sport, exercise and health the physiological responses of $\mathrm{VbX}$ remain equivocal because a number of studies have used various protocols, of different methods of application, vibration parameters, training duration and exercises performed with vibration. Some recent $\mathrm{VbX}$ reviews have been conducted on 
specific population groups such as trained $[22,133]$, and untrained [97] but they have not integrated acute, short- and long-term effects of $\mathrm{VbX}$ on reflex muscle activity, cardiovascular responses and muscle function for various populations of healthy, and compromised health. Therefore, the purpose of this review was to critically examine the physiological effects of VbX, namely reflex activity, muscle function, and cardiovascular indices and to elucidate its potential use for those with compromised health where safety aspects and loading parameters of VbX are also discussed.

\section{Method}

$\nabla$

A search was conducted using electronic databases Medline, PubMed, ISI Web of Knowledge and Scopus using key words of vibration and whole-body vibration, in combination with exercise, training, power, force, strength, cardiovascular, blood flow, heart rate, reflex, electromyography, Multiple Sclerosis, stroke, Parkinson's Disease, postmenopausal and elderly. Articles were checked for relevant content and were included from the following 1. published in English; 2. examined acute (single session), short-term (multiple sessions performed up to 2 months), and long-term (multiple sessions greater than 2 months) effects; 3. Status of participants included healthy, trained, untrained and specific health ailments and Conference abstracts and proceedings were not included.

\section{Vibration exercise}

\section{Types of vibration exercise}

VbX has taken on many different forms. Small vibratory units have been placed directly on the muscle or tendon $[62,132]$ and larger custom built units have been constructed for flexibility training $[112,113]$. Vibrating units have also been attached to resistance training equipment to elicit vibration transmission through the cables of various machines [59]. Currently, there are
2 commercial forms of vibration platforms manufactured for the health and fitness industry. The first type of platform (e.g. Galileo ${ }^{\circledR}$ ) has a teeterboard that produces side-alternating vertical sinusoidal vibration (SV) to the body. It rotates around an anteroposterior horizontal axis, so when the feet are further from the axis it results in a larger vibration amplitude ( $\boldsymbol{0}$ Fig. $\mathbf{1}$ ).

The side-alternating movement is asynchronous where the unilateral vibration is applied alternately to the left and right foot. The other commercial machines (Power Plate ${ }^{\circledR}$, Nemes ${ }^{\circledR}$, Vibra Pro $^{\circledR}$,Vibrafit ${ }^{\circledR}$, Fit Vibe $^{\circledR}$, Pneu-vibe ${ }^{\circledR}$, Vibrogym $^{\circledR}$, Soloflex ${ }^{\circledR}$, Bodypulse ${ }^{\circledR}$, Juvent $1000^{\circledR}$ ) produce vertical synchronous vibration (VV) where both legs are vibrated as the platform moves predominately in the vertical direction. This results in simultaneous and symmetrical movement of both sides of the body during the exposure ( $\bullet$ Fig. 1 ). Hand-held powered vibrating dumbbells have also been commercially manufactured for exercising the upper-body (Galileo TOP $^{\circledR}$, Mini-VibraFlex ${ }^{\circledR}$ ) where the central handle piece of the dumbbell rotates and produces oscillatory movements to the body of varying frequencies $(0-30 \mathrm{~Hz})$.

The debate over which platform is superior is currently equivocal. Research performed by Abercromby et al. [1] reported that the lower limb extensors (vastus lateralis and gastrocnemius) were activated significantly more during SV than VV from an acute exposure; however, the activation of the tibialis anterior was significantly greater during VV than SV. Furthermore, during dynamic (from $10^{\circ}$ to $35^{\circ}$ of knee flexion, at a tempo of $4 \mathrm{~s}$ up $4 \mathrm{~s}$ down) and static squatting ( $18.5^{\circ}$ knee flexion), SV produced a greater activation of the lower limb muscles compared to VV. In a later study, the same authors [2] reported that across different knee angles $\left(5-35^{\circ}\right)$ vibration transmitted to the upper-body and head was $71-189 \%$ greater during VV than SV. The authors concluded that during SV the pelvis damps the vibration energy more than the VV. Earlier, Rittweger et al. [104] had proposed a similar hypothesis: in SV the feet are alternated between up and down positions, causing rotation of pelvis and flexion of the spinal column, which decreases the vibration

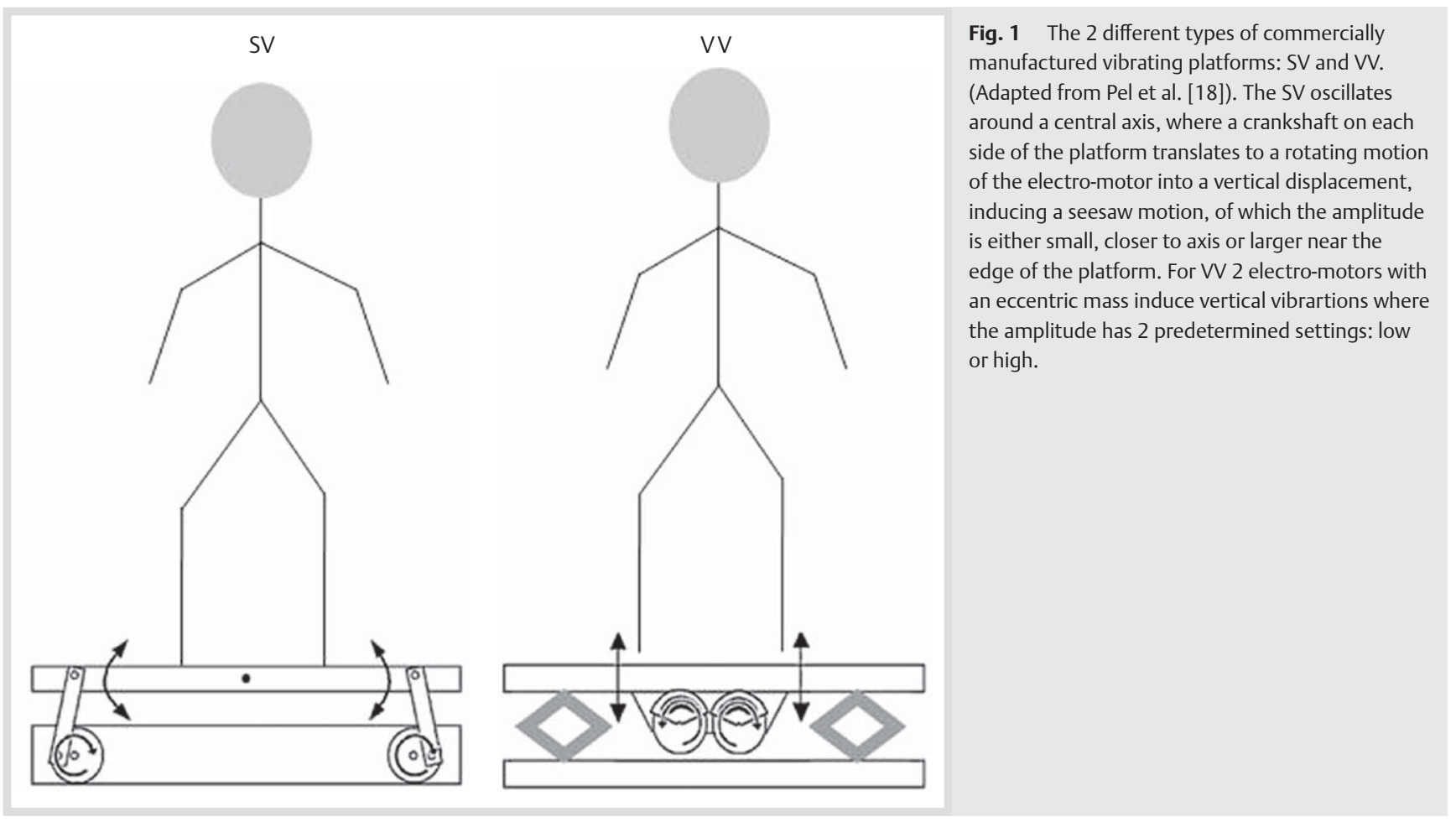




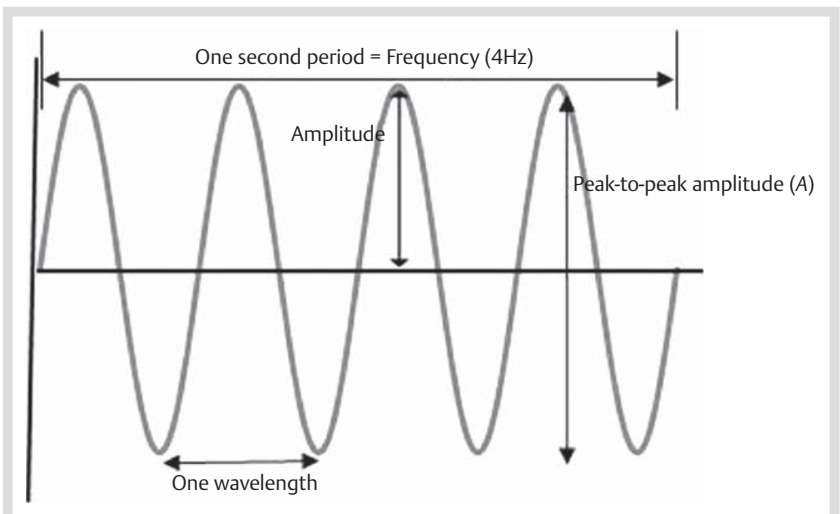

Fig. 2 Parameters of sinusoidal oscillation.

transmission to the head. However, no kinematic analyses have been performed on SV and VV to validate this claim. It should be noted that in Abercomby et al.'s [1] acute study the dynamic squat was only performed through a limited range of knee flexion $\left(10-35^{\circ}\right)$.

\section{Transmission of vibration exercise}

The majority of contemporary vibration machines produce periodic sinusoidal oscillations, where energy is transferred from the vibratory machine to the human body. The vibratory load is dependent on 4 parameters: frequency, amplitude, acceleration, and duration. The number of cycles of oscillation determines the frequency; the amplitude refers to the displacement of the oscillatory motion ( $\bullet$ Fig. 2 ); the acceleration $\left(\mathrm{m} / \mathrm{s}^{2}\right.$ or $g$ ) determines the magnitude; and duration refers to the exposure time. Normally, $\mathrm{VbX}$ is administered in the range of $0-45 \mathrm{~Hz}$, amplitude of $0-12 \mathrm{~mm}$ (peak-to-peak amplitude) and peak acceleration of $0-18 \mathrm{~g}$.

The motion of vibration training is sinusoidal. The acceleration transmitted to the body is based on the principle of peak acceleration $(a)$ being the product of angular frequency $(\omega)$ squared and peak-to-peak amplitude $(A)$, where $A$ is converted from $\mathrm{mm}$ to m. (Equation 1)

$$
\begin{array}{ll}
a_{\text {peak }}=\omega^{2} \mathrm{~A} & \left(\mathrm{~m} / \mathrm{s}^{2}\right) \\
\omega=(2 \pi f) & (\mathrm{Hz})
\end{array}
$$

Angular frequency $(\omega)$ is the product of $2 \pi$ and vibration frequency $(f)$ (Eq. 2). Where $f$ is the number of vibratory cycles per unit of time. Therefore, $1 / f$ provides the duration of a single cycle, $\mathrm{Hz}$, which is equivalent to cycle per s. Thus, $\omega$ is proportional to $f$ as given by $2 \pi f, \mathrm{~Hz}$, which is equivalent to cycle per s. For example, a vibration frequency of $26 \mathrm{~Hz}$ and peak-to-peak amplitude of $6 \mathrm{~mm}$ would produce a peak acceleration of $160 \mathrm{~m} / \mathrm{s}^{2}(16.3 \mathrm{~g})$. Changes in vibration frequency or amplitude will determine the changes in acceleration transmitted to the body. The greatest acceleration (or gravitational load) is high when both frequency and peak-to-peak amplitude are at their maximum. For example, a vibration frequency of $26 \mathrm{~Hz}$, at a peak-to-peak amplitude of $6 \mathrm{~mm}$, will produce a peak angular acceleration of $160 \mathrm{~m} / \mathrm{s}^{2}$ or $16.3 \mathrm{~g}$. The vibration frequency and amplitude can be manipulated to determine the desired peak acceleration; however, only a few studies have investigated the dose relationship on neuromuscular and performance aspects. Damping and stiffness adjustments are 2 other factors that need to be considered when vibration is transmitted from the vibratory device to the human body. Wakeling et al. [131] have reported that vibration is likely to be damped by tissues and fluids where the mechanical energy is absorbed by structures, leading to heat generation.

Anecdotal reports claim that standing erect, as opposed to squatting, evokes a stronger transmission of the vibration to the head, and shifting body weight to the forefront of the foot will reduce the vibration transmission. Lafortune et al. [70] confirmed this, reporting that when the knees were extended the vibration frequency above $10 \mathrm{~Hz}$ was more effective in transmitting to the hip compared to a frequency of less than $5 \mathrm{~Hz}$. Rubin [109] found that at low vibration frequency the vibration transmission to the hip was dissipated; however, when the frequency was raised to $15-35 \mathrm{~Hz}$, a higher response at the hip was evident. A scientific model [134] has been used to describe the transmission of vibration; however, this model has lacked the inclusion of the 3 lower limb joints (ankle, knee and hip) and the researchers have not considered the effect of the range of joint motion on vibration transmission. Abercromby et al. [1] stated that the damping of mechanical energy by the legs depends not only on the compliance of ankle, knee, and hip joints, but also on the modulation of leg muscle activation. These researchers found that the greatest mechanical impedance (as determined by a decrease in joint compliance and an increase in the absorption of vibration energy) occurred at a knee angle of $10-15^{\circ}$. They also found that acceleration of the head decreased as knee angle increased from 10 to $30^{\circ}$, and was greater in acute VV than SV $(f=30 \mathrm{~Hz}$, $A=4 \mathrm{~mm})$. The authors concluded that squatting at a knee angle of $26-30^{\circ}$ dissipated head vibration and that the use of small knee flexion angles during VbX increased the likelihood of negative side effects as the greatest mechanical energy is likely to be transmitted to the upper-body and head and should, therefore, be avoided.

\section{Parameters of vibration exercise}

\section{Frequency}

Prior to the inception of commercialised VbX, vibration studies focused on high vibration frequency; vibratory units were applied directly to the muscle or tendons of animals or humans for a very short duration. McCloskey et al. [80] applied direct acute vibration of $100-200 \mathrm{~Hz}$ to the hindlimb of a cat's tricep surae muscle, and Bongiovanni and Hagbarth [11] applied 2 min of direct vibration of $150 \mathrm{~Hz}$ to human tendon ankle dorsiflexors to stimulate the tonic vibration reflex (TVR). It is not exactly known how the scientific merit of low frequency vibration was validated, but Nazarov and Spivak [86] may have had a part in determining it. They used an arbitrary frequency of $23 \mathrm{~Hz}$ because they feared that the vibration would disappear during transmission in the tissue if a higher frequency was selected. Another explanation states that from a natural frequency lower limb muscles respond between a range of $5-65 \mathrm{~Hz}$ [87]. There is little scientific documentation on the appropriate vibration frequency ( $f$ ); however, Bosco et al. [13] exposed handball and waterpolo athletes to 10 days of intermittent vibration $(f=26 \mathrm{~Hz}$, SV, Galileo) and reported an increase in vertical jump height by $12 \%$, but gave no rationale as to why $26 \mathrm{~Hz}$ was selected. In a follow-up study, Bosco et al. [14] confirmed their earlier findings that acute vibration performed at $26 \mathrm{~Hz}$ on an SV (Galileo) platform had a positive effect on muscular performance by shifting the force-velocity and power-force relationship to the right, enhancing average force, velocity and power in a sample size of 

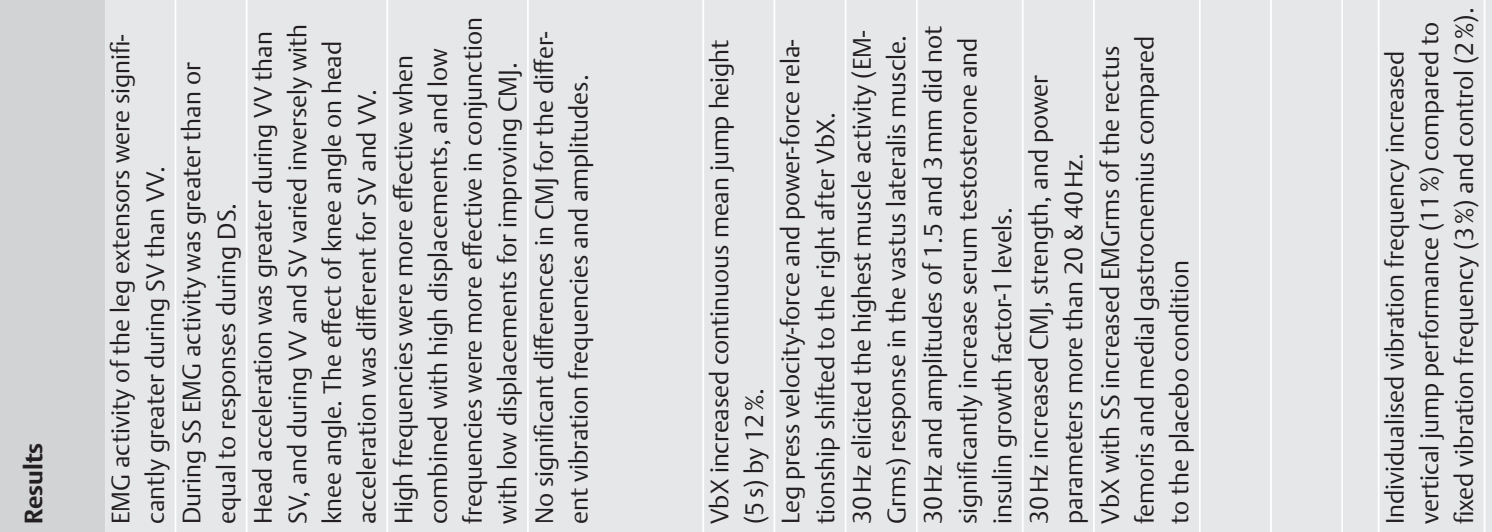

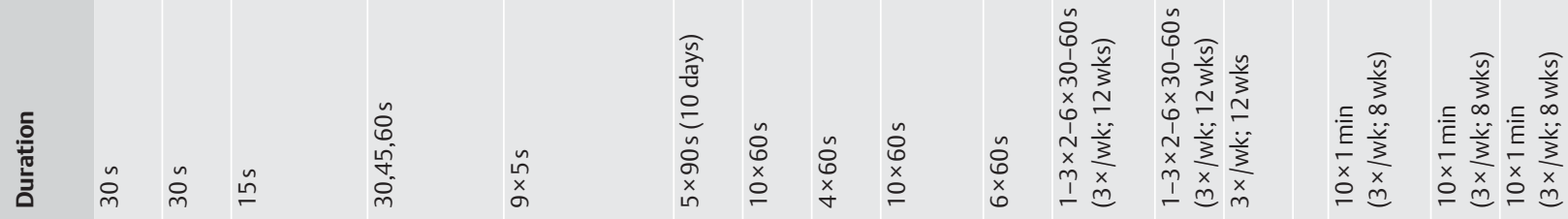

$\frac{\sqrt{0}}{\frac{\pi}{2}}$

$\stackrel{\infty}{2} \frac{\infty}{2} \quad \frac{o}{2}$

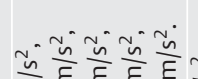

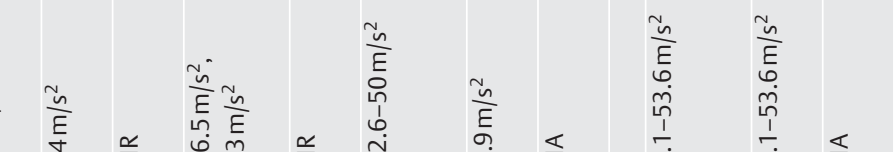

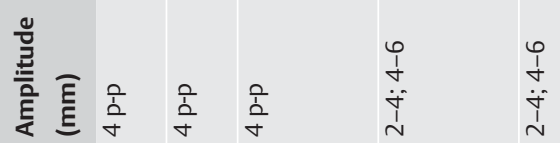

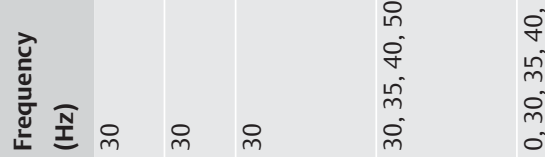

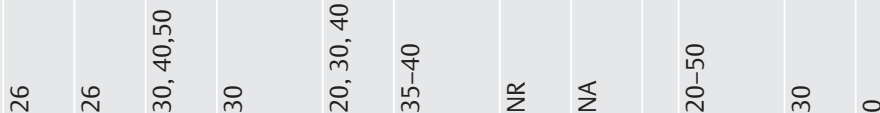

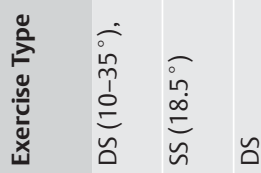

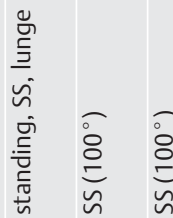

$\frac{\mathscr{Q}}{\infty}$ 岳

$\widehat{0}$

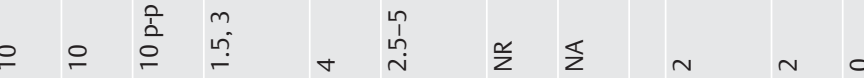

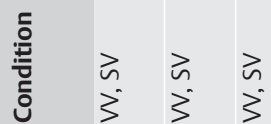

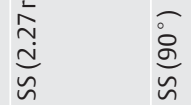

告

离离

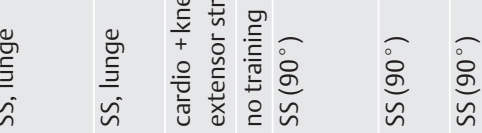

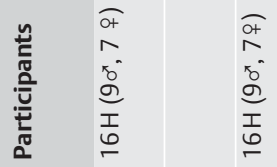

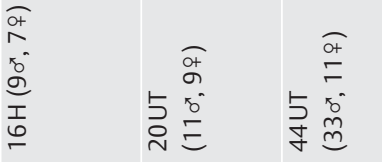

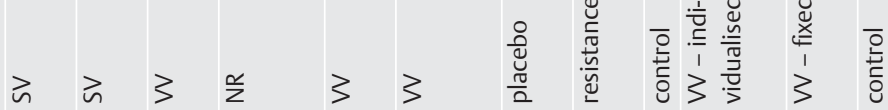

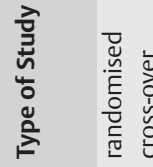

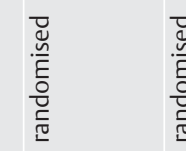

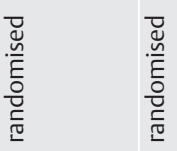

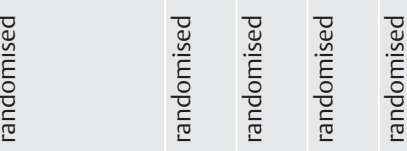

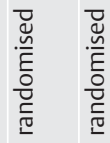

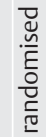

童

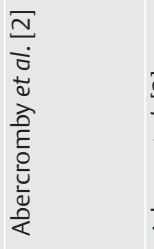

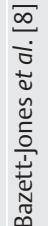

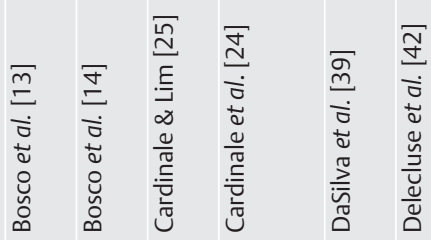

胥 


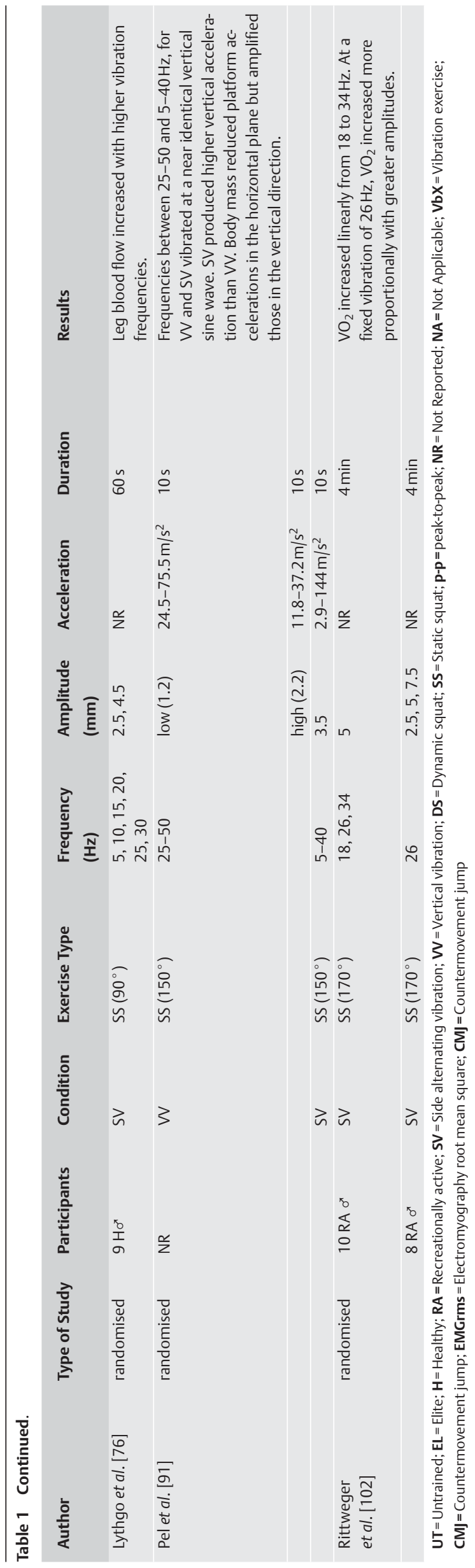

6 elite volleyball players. Akin to their previous study, no rationale was given for the selection of the vibration frequency.

It took Cardinale and Lim [25] to provide some insight on the optimal vibration frequency. Using electromyography (EMG) to validate the vibration frequency, these authors found that the EMG response of the vastus lateralis, as analysed by the root mean square (rms) was significantly higher in acute $30 \mathrm{~Hz}$ compared to 40 and $50 \mathrm{~Hz}$ when standing in a half squat position (knee angle $100^{\circ}$ ) on a VV (Nemes) platform for $60 \mathrm{~s}$ (॰ Table 1 ). Delecluse et al. [43] also reported that standing in a static half squat position on a vibrating platform $(f=35 \mathrm{~Hz}$, Amplitude $(A)=5 \mathrm{~mm}$, acceleration $(a)=3.9 \mathrm{~g})$ increased $\mathrm{EMG}_{\mathrm{rms}}$ of the rectus femoris and medial gastrocnemius muscles compared to the placebo condition $(a=0.4 \mathrm{~g})$ after 12 weeks of VbX. However, both Delecluse et al.'s [43] and Cardinale and Lim's [25] studies were conducted on a VV platform, with no comparison being made to a SV machine.

Recently, a number of studies ( $\odot$ Table 1 ) have examined whether a dose relationship exists between different vibration frequencies and muscular performance, where the majority have used vertical jump (VJ) height as the performance measure. Da Silva et al. [39] found that an acute intermittent vibration protocol performed at a frequency of $30 \mathrm{~Hz}(A=4 \mathrm{~mm})$ increased VJ height and leg power compared to $20 \mathrm{~Hz}$ and $40 \mathrm{~Hz}$. However, this study lacked a control condition; the body posture during vibration was not described; and no measures of EMG were collected. Likewise, Bazett-Jones et al. [8] reported a significant increase of $9 \%$ and $8.3 \%$ in $\mathrm{VJ}$ height in young untrained women when acutely exposed to $40 \mathrm{~Hz}$ and $50 \mathrm{~Hz}$ compared to the control condition, but there was no increase inVJ height for the untrained men. However, the small sample size $(n=11)$ of the women compared to the men $(n=33)$ may have incurred a type I error, and the vibration exposure time of $45 \mathrm{~s}$ may have been insufficient to elicit the required neuromuscular responses for the males.

VbX is like any other form of training - every individual will have a response to an optimal intensity, and/or training load. In physical conditioning, most fitness programmes consider individual responses, and are tailored to suit the individual; however, in $\mathrm{VbX}$ there has been a tendency for one vibration frequency to be used by all. Until recently, this was the status quo; however, Di Giminiani et al. [44] observed that after 8 weeks ( $3 \mathrm{x} /$ week) of training with the vibration frequency individualised by determining the $\mathrm{EMG}_{\mathrm{rms}}$ activity of the vastus lateralis performed in a half squat position, the mean power of squat jump and jump height from a continuous rebound jumping test increased $11 \%$ and $18 \%$ respectively in comparison to the fixed $(30 \mathrm{~Hz})$, or no vibration groups. These results clearly indicate that vibration frequency should be individualised to fully maximise the benefits of vibration training. Just as other fitness and resistance programmes are individualised for the client, vibration training should follow suit. However, it is unknown if $\mathrm{EMG}_{\mathrm{rms}}$ is the most appropriate measure to individualise each person's optimal vibration frequency. It may not be practical, as EMG equipment is often expensive, and may not be affordable or readily available in practical settings, such as clinics and gyms. It also requires a certain level of expertise. Further, it should be noted that in Di Giminiani et al.'s [44] study only one site (vastus lateralis) was used to record EMG activity. Other muscles, such as the triceps surae and hamstring group, need to be analysed before a conclusion can be reached that the use of one particular muscle will provide the optimum vibration frequency. 


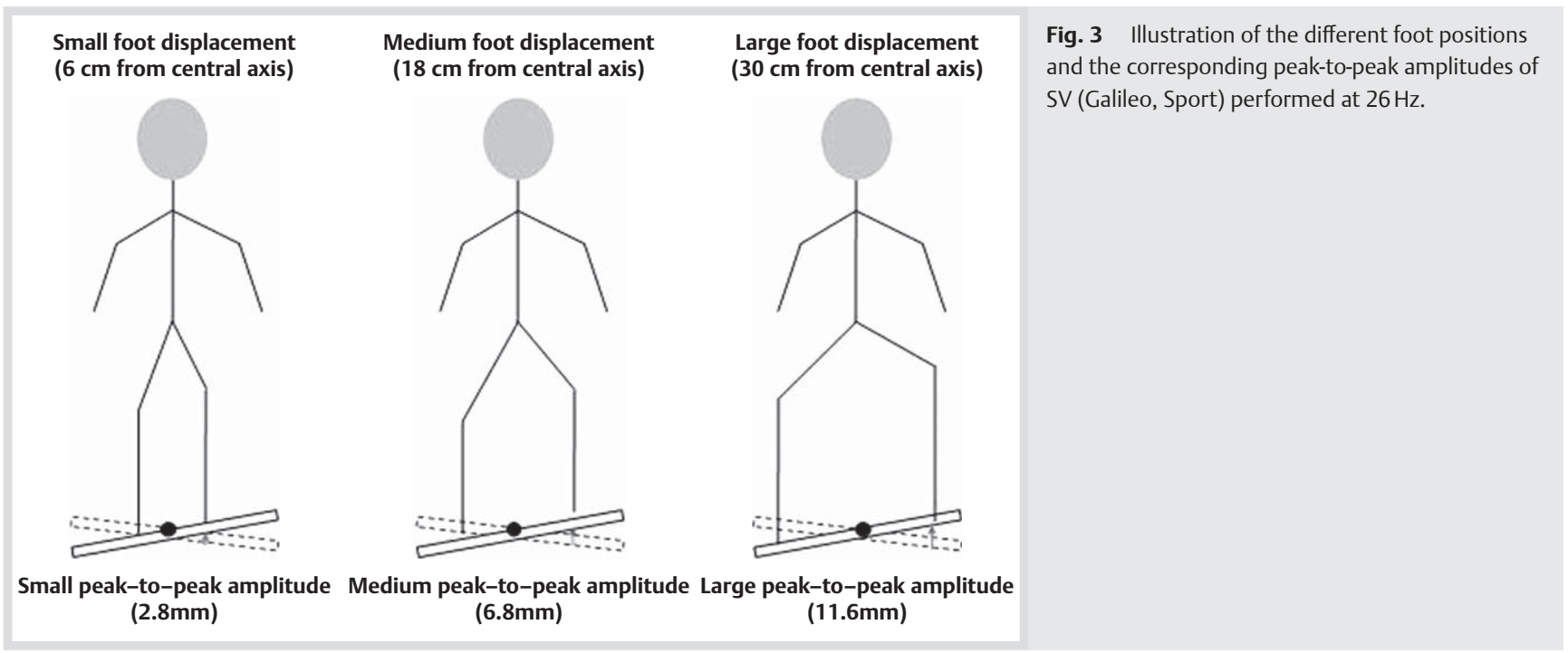

\section{Amplitude}

Amplitude is defined as the maximum displacement of a vibration point from a mean position, compared to peak-to-peak amplitude $(A)$, which is referred to as the height from the lowest to highest vibration wave ( $\bullet$ Fig. 2 ). For SV, the amplitude is dependent on where the feet are placed on the platform. When the feet are close to the middle of the platform it equates to a small peak amplitude $(\sim 3 \mathrm{~mm})$. A wide stance equates to a greater amplitude $(\sim 12 \mathrm{~mm})$ ( $\bullet$ Fig. 3 ). This differs to VV, where foot placement is independent of amplitude and has a pre-setting of $0-2 \mathrm{~mm}$ or $4-6 \mathrm{~mm}$. Using an acute intermittent vibration protocol Cardinale et al. [24] found no differences in insulin growth factor 1 (IGF-1) and testosterone levels when participants were exposed to high amplitude $(A=3 \mathrm{~mm})$, low amplitude $(A=1.5 \mathrm{~mm})$ and zero amplitude $(A=0 \mathrm{~mm})$ at a fixed vibration frequency $(f=30 \mathrm{~Hz})$. The authors failed to document the type of vibration platform; therefore, if a VV was used the selection of a small amplitude may not have elicited the required response. Using a SV (Galileo) platform where participants stood in an upright stance $\left(10^{\circ}\right.$ knee flexion) at a fixed frequency of $26 \mathrm{~Hz}$, Rittweger et al. [102] reported that acute $\mathrm{VbX}$ increased the oxygen cost increased in all 3 amplitudes $(2.5,5,7.5 \mathrm{~mm})$ compared to baseline levels, with the highest amplitude $(7.5 \mathrm{~mm})$ having the greatest oxygen cost $(7.3 \mathrm{ml} / \mathrm{kg} / \mathrm{min}$ compared to resting $3.6 \mathrm{ml} / \mathrm{kg} / \mathrm{min}$ ). For muscular power, Adams et al. [3] showed that acute high vibration frequency $(50 \mathrm{~Hz}, \mathrm{VV}$, [Power Plate]) with high amplitude $(4-6 \mathrm{~mm})$, and low frequency $(30 \mathrm{~Hz})$ with low amplitude (2-4 mm) were effective for increasing $\mathrm{VJ}$ power. Moreover, Lythgo et al. [76] observed that low vibration amplitude ranging from $2.5 \mathrm{~mm}$ to $4.5 \mathrm{~mm}$ (SV) was able to elicit an increase in mean blood cell velocity by $27 \%$, when acute vibration frequency was progressively increased from 5 to $30 \mathrm{~Hz}$.

To date, no study has directly compared the different amplitudes between the VV and SV platforms. As discussed earlier, the placement of the feet determines the amplitude in SV, which may affect the transmission of vibration to the various regions of the body. There has been some speculation that different body masses may alter the amplitude of vibration platforms; a heavier mass may decrease the amplitude of the platform. This presumption has been challenged based on recent evidence from Pel et al. [91] who reported no change in amplitude when SV (Galileo) and VV (Power Plate) platforms were loaded with 2 dif- ferent body masses. The authors observed that the acceleration (g) from acute vibration in the vertical direction of the SV platform was reduced when vibration frequency was increased from 30 to $40 \mathrm{~Hz}$. However, only 2 different body masses $(62 \mathrm{~kg}$ and $81 \mathrm{~kg}$ ) were used in this study and the authors did not consider that many athletes and overweight individuals that use VbX would be outside this body mass range. Consequently, the range is insufficient to critically discuss the effect of body mass on the frequency of $\mathrm{VbX}$. Additionally, the interaction of body mass and stance (knee angle) on amplitude requires further investigation. A current criticism of the literature is that most studies do not provide such details on how amplitude is calculated to whether it is measured by an accelerometer or computed by a mathematical equation. Much confusion surrounds the terms amplitude, peak-to-peak amplitude, and displacement because they are used interchangeably and standardisation of terminology is required. It is advised that a consensus be established in an attempt to gain consistency between research protocols. Lorenzen et al. [73] recommended that the term 'peak-to-peak amplitude' be used, and for vibrating platforms an anatomical landmark, such as the middle toe of the foot, be used to standardise the amplitude measurement, which needs be measured and reported.

\section{Acceleration}

VbX generates mechanical vibration resulting in acceleration, which is the product of angular velocity $(2 \pi f)^{2}$ and amplitude $(A)$, where it has also been coined magnitude and the unit is either expressed as $\mathrm{m} / \mathrm{s}^{2}$ or as multiples of terrestrial gravitation in $g$ (where $1 g=9.81 \mathrm{~m} / \mathrm{s}^{2}$ ). Acceleration is proportional to the force applied. Therefore, increasing acceleration relies on changing the frequency and amplitude to increase acceleration of vibration being transmitted to the body [26], which is similar to adding extra load in conventional resistance training $(F=m a)$. $\mathrm{VbX}$ relies on increasing acceleration to increase force, where the force is likely to be the primary stimulus to promote changes within the body. However, as the vibrations travel through the body the effect of the force is likely to be damped by muscles, tissues and fluids [131]. A high level of acceleration can be potentially harmful but with careful measurement the optimal training stimulus can be monitored, which is important for making comparisons with other studies. 
A large number of acute, short- and long-term $\mathrm{VbX}$ studies have reported acceleration but have not documented how they measured or calculated it $[14,15,23,24,48,59,67,101,110,123,124]$ . A few studies that have directly measured the acceleration source by securing accelerometers to vibration platforms $[8,42-$ $44,76,91,105]$, and have reported mean accelerations of $2.2 \mathrm{~g}$ $(f=30 \mathrm{~Hz}, A=2-4 \mathrm{~mm}, \mathrm{VV}), 5.8 \mathrm{~g}(f=50 \mathrm{~Hz}, A=2-4 \mathrm{~mm}, \mathrm{VV})[8]$ $2.5 g$ to $7.7 g(f=25-50 \mathrm{~Hz}, A=$ high, VV), $0.3 g$ to $14.7 g(f=5-40 \mathrm{~Hz}$, $A=$ high, SV) [91] , and $0.1 \mathrm{~g}$ to $5.5 \mathrm{~g}(f=20-55 \mathrm{~Hz}, A=2-\mathrm{mm}, \mathrm{VV})$ [44]. Additionally, some researchers have placed accelerometers on body landmarks to determine the acceleration transmission of the vibration that passes through the various joints $[2,37]$ and found that greater accelerations were observed proximal to the vibration platform compared to distal locations. According to Lorenzen et al. [73] all studies should document maximum acceleration $\left(\mathrm{m} / \mathrm{s}^{2}\right.$ or $\left.g\right)$ and provide an explanation of how peak or mean acceleration was determined.

\section{Duration}

Duration refers to the exposure time to vibration. Most VbX studies have either been performed acutely for single or multiple sessions, either intermittently (30-60 s exposure) or continuously (3-5 min) $[13,14]$. It appears that Bosco's [120] acute intermittent protocol of 10 repeated exposures of 1 min interspersed with 1 min rest has been a common duration used in subsequent vibration studies, but with little justification. Currently, there is little scientific evidence on what the optimal duration is for intermittent and continuous sessions. Nevertheless, Adams et al. [3] found no significant differences in vertical jump peak power when untrained participants were exposed to acute vibration durations of $30 \mathrm{~s}, 45 \mathrm{~s}$, or $60 \mathrm{~s}(f=30-50 \mathrm{~Hz} A=$ 2-4, 4-6 mm). Moreover Stewart et al. [120] reported that standing $\left(5^{\circ}\right.$ knee flexion) on a SV, isometric peak torque increased by $3.8 \%$ after 2 min of continuous vibration, compared to decrements in peak torque at 4 and $6 \mathrm{~min}(f=26 \mathrm{~Hz}, A=4 \mathrm{~mm})$. In longterm vibration training studies, various exposure times have been reported for studies conducted over 6-12 weeks and 3-8 months $[1,2,14,15,18,24,25,40,45,48,57]$. There seems to be a knowledge deficit regarding the ability of continuous $\mathrm{VbX}$ to potentiate performance measures. As with resistance training, there may be a point of diminishing returns with respect to the duration of $\mathrm{VbX}$. Durations of greater than $1 \mathrm{~min}$ are likely to either involve lower levels of acceleration (and potential for adaptations in strength and power) or greater injury risk if high duration and accelerations are combined. Therefore, intermittent protocols may be preferred to continuous exposure because it stimulates muscle while limiting fatigue. However, more work is required to determine whether there is an optimal duration exposure time for $\mathrm{VbX}$ in acute and long-term studies.

\section{Exercises and posture}

Static and dynamic squats are common exercises performed on vibration platforms [13,32,77,123]; however, a combination of lower and upper-body exercises have also been performed on various vibrating platforms [1].

The knee joint angle is a critical factor when performing a static or dynamic squat on a vibrating platform. Abercromby et al. [1] reported that a static squat at $18.5^{\circ}$ provided greater muscle activation compared to dynamic squatting ( $10-35^{\circ}$ knee angle). Additionally, these authors observed that during dynamic squatting, $\mathrm{EMG}_{\mathrm{rms}}$ of vastus lateralis, gastrocnemius, and tibialis anterior activity were higher in smaller $\left(10-15^{\circ}\right)$ compared to larger flexion angles $\left(31-35^{\circ}\right)$ for acute exposures on both SV and VV platforms. Caution should be given to Abercromby et al.'s [1] findings, as a narrow range of knee angle $\left(10-35^{\circ}\right)$ was tested during the dynamic squat and only one fixed angle was assessed for the isometric squat position $\left(18.5^{\circ}\right)$. Further, it is unknown whether greater knee angles would continue to decrease muscle activity in dynamic squatting, and it is unknown what effect EMG activity has on high $\left(120^{\circ}\right)$ or low $\left(90^{\circ}\right)$ isometric squats. It remains equivocal whether a greater knee angle elicits a decrement in EMG activity for dynamic and static squatting, and if amplitude or frequency provides the greatest stimulus for muscle activity change and where the exposure duration influences this as well. Roelants et al. [107] reported that during VV ( $f=35 \mathrm{~Hz}, A=2.5 \mathrm{~mm}$ ) EMG was significantly higher in single leg squat (knee angle $125^{\circ}$ ) compared to bilateral squat at $90^{\circ}$ and $125^{\circ}$ knee angle. Using 2 knee flexion angles of $10^{\circ}$ and $70^{\circ}$ $(f=20 \mathrm{~Hz}, A=5-9 \mathrm{~mm}, \mathrm{SV}$, [Galileo 900]) Savelberg et al. [114] reported that after 4 weeks ( $3 \mathrm{x} /$ week) of $\mathrm{VbX}$ the $10^{\circ}$ knee angle shifted to a more extended knee joint angle. In contrast, the larger knee angle $\left(70^{\circ}\right)$ shifted to a more flexed knee joint position, and the authors concluded that vibration caused a change in muscle length which shifted the knee angle. However, to confirm this finding a control condition should have been included. Therefore, future research needs to focus on different isometric knee angles in response to larger knee angles in dynamic squatting and its application to muscle performance.

\section{Safety of vibration exercise}

Occupational vibration can be detrimental to one's health, especially for workers who are constantly and continually exposed to vibrations from different types of machinery [82]. However, most exercise vibration studies are conducted acutely and intermittently with no incidences of ill-effects having being reported. Conversely, Crewther et al. [37] observed that untrained participants exposed to acute vibration frequencies $(10,20,30 \mathrm{~Hz})$, amplitudes (1.25, 3, $5.25 \mathrm{~mm}$ ) and postures (standing, squat) suffered from side-effects, such as hot feet, itching of the lower limbs, vertigo and severe hip discomfort. Likewise, Cronin et al. [38] reported that untrained participants suffered from vibration pain of jaw, neck and lower limbs from an acute intermittent VbX protocol $(f=26 \mathrm{~Hz} ; A=6 \mathrm{~mm})$, which subsided after 7-10 days of physiotherapy treatment. However, Crewther et al. [37] and Cronin et al. [38] did not fully disclose how the participants were familiarised on the vibration platform, or whether they screened the participants for vibration side-effects. Both studies required the participants to slightly flex their knees; this small knee angle may have increased the vibration transmission to areas such as the head or hip. Recent research has concluded that the smaller the knee angle, the greater the vibration transmission to the head $[1,91]$. The findings of Crewther et al. [37] and Cronin et al. [38] are very uncommon, but highlight the need for researchers and exercise specialists to be fully trained on the use of vibration technology before participants take part in vibration led exercise, research and rehabilitation programmes.

Additionally, it has been reported by Rittweger et al. [101], Kerschan-Schandl et al. [67], Russo et al. [110], Roelants et al. [106], Hazell et al. [54], and Broadbent et al. [16] that acute VbX can elicit erythema of the lower limbs, with anecdotal reports from participants suggesting that $\mathrm{VbX}$ causes a hot sensation of the legs and acute itchiness, which normally subsides within minutes of $\mathrm{VbX}$ and has no deleterious effect on the body. 
However, it is unknown what causes the itchiness; one proposal suggests that the increase in blood flow is the main contributor, while another thought is that vibration induces skin shear forces which promotes vasodilation and is mediated by release of histamine [100]. Broadbent et al. [16] has postulated that acute VbX may cause an excitatory response on mast cells to produce histamine, which causes vasodilation and promotes erythema itchiness. Results from their study, however, indicate that histamine levels were lower in the leg receiving vibration $(f=40 \mathrm{~Hz}$, $A=5 \mathrm{~mm}, \mathrm{VV}$ ) after muscle damage was elicited from downhill running, compared to those with muscle damage who received no vibration. Further, they speculate that the increase in blood flow from the vibration could have increased the clearance rate of histamine. Therefore, the mechanism causing erythema remains unidentified, and warrants further investigation.

The effects of vibration exercise on reflex activity and Jendrassik manoeuvre

Tendon reflex (stretch reflex)

Rittweger et al. [103] reported an enhancement of the patellar tendon stretch reflex following acute $\operatorname{VbX}(f=26 \mathrm{~Hz}, A=6 \mathrm{~mm}, \mathrm{SV}$, [Galileo]) with dynamic squatting to exhaustion, and suggested that $\alpha$-motoneurons were augmented by the vibration, which recruited high-threshold units and muscle fibres. However, no effect on patellar tendon reflex has been reported after acute intermittent ( $f=26 \mathrm{~Hz}, A=4 \mathrm{~mm}, \mathrm{SV}$, [Galileo]) [57] or continuous [33] ( $5 \mathrm{~min})(f=26 \mathrm{~Hz}, A=6 \mathrm{~mm}, \mathrm{SV}$, [Galileo]) vibration exposure. Recently Melnyk et al. [81] elicited the stretch reflex in the hamstrings by inducing an anterior tibial translation during standing, and compared the reflex response between a control and acute intermittent vibration group $(f=30 \mathrm{~Hz}, A=4 \mathrm{~mm}, \mathrm{VV}$, [Power Plate]). The researchers found post-vibration, anterior tibial translation displacement decreased with a corresponding increase in EMG of the hamstring short latency response. The authors concluded that the effect of increased knee stability was caused by reflex excitability. However, from this study it is not conclusive whether the stretch reflex was potentiated from acute $\mathrm{VbX}$ and it remains speculative whether spinal reflexes are the main mechanism.

\section{Hoffmann reflex (H-reflex)}

The Hoffmann reflex (H-reflex) is a measure of assessing monosynaptic activity of the spinal cord [93]. The H-reflex differs to the tendon reflex because it is activated by electrical rather than mechanical stimulation, which bypasses the muscle spindle by acting directly on the afferent fibre. The electrical stimulus causes a corresponding H-reflex, which is the result of increased excitability of the Ia afferent and $\alpha$-motoneurons. As the strength of the electrical stimulus increases, the efferent fibres become excited and induce a direct muscle contraction, known as the M-response. Further increase in the stimulus strength results in suppression of the H-reflex but M-response becomes augmented.

Thompson and Belanger [121] reported that self-paced inline skating for 35 min elicited a mean vibration frequency of $141 \mathrm{~Hz}$ from the skate chassis and $34 \mathrm{~Hz}$ from the middle portion of the tibia, which resulted in the H-reflex being suppressed by $35 \%$ compared to resting conditions. The authors concluded that presynaptic inhibition was the main factor for suppressing the reflex response. Similarly, Armstrong et al. [5] found the H-reflex was suppressed in the first minute post-vibration after a single minute bout of VV $(f=40 \mathrm{~Hz}, A=2-4 \mathrm{~mm})$. But earlier work from
Nishihira and associates [88] found that post-vibration the $\mathrm{H}$-reflex and $\mathrm{H}$ max/M max ratio was enhanced, suggesting that motoneuron excitability was heightened. The dissonance between Nishihira and associates [88] and Armstrong et al.'s [5] findings is probably due to the different protocols. Nishihira et al. [88] used a $3 \mathrm{~min}$ exposure performed on SV platform $(f=25 \mathrm{~Hz})$ where the H-reflex was elicited from a seated position, which differs to Armstrong et al.'s [5] 1 min duration performed on $\mathrm{VV}(f=40 \mathrm{~Hz})$ with the H-reflex elicited from a supine position.

\section{Jendrassik manoeuvre}

The Jendrassik manoeuvre involves contracting remote muscles, normally of the upper-body (particularly the forearm and jaw muscles) to induce a reflex response. A common method for eliciting a reflex is to grasp the hands and pull them apart - this potentiates the stretch reflex and H-reflex. In the clinical setting, the Jendrassik manoeuvre has been used to induce a full-sized reflex in neurologically impaired patients. In young (25yrs) and older (75yrs) healthy people directly applied vibration to the quadriceps $(f=100 \mathrm{~Hz}, A=2 \mathrm{~mm})$ produced a post-vibration decrease in patella tendon reflex force, but the younger group showed a greater reflex inhibition [50]. Moreover, the effect of the Jendrassik manoeuvre facilitated the patella tendon reflex more in young people than in the older group ( $97 \%$ vs. $64 \%$ ). The authors concluded that the age-related changes were due to changes in pre-synaptic inhibition pathways and motoneuron input resistance, confirming that the integrity of the spinal interneuronal pathways deteriorate with ageing [19]. The reduction in muscle vibration inhibitory effect of older adults suggests that the pre-synaptic inhibition of Ia afferents may deteriorate with an increase in age, or the aged muscle spindle may reduce the number of Ia afferents activated by the vibratory stimulus. Recently, Cochrane et al. [31] reported that superimposing the Jendrassik manoeuvre upon acute VBX enhanced metabolic rate in old and young people but there were no significant differences in the elevated metabolic rate between the groups.

To date, there is a lack of research on how $\mathrm{VbX}$ may influence spinal reflexes but there remains a positive belief that spinal reflexes are responsible for $\mathrm{VbX}$ potentiating effects. However, in light of recent research it remains equivocal whether the spinal reflexes are the major contributor because blood flow, muscle temperature, and other neural aspects increase as a result of VbX. Additionally, it has been difficult to ascertain if spinal reflexes are potentiated from $\mathrm{VbX}$ due to different vibration methods, parameters (amplitude, exposure duration, rest interval), prescription (number of repetitions, sets, exercises) and participant characteristics (body/muscle mass, training status, age, gender). In the future, this needs to be standardised to determine if $\mathrm{VbX}$ influences spinal reflexes.

\section{The effects of vibration exercise on muscle activity Lower body}

It is possible to measure electromyography (EMG) activity without any artefacts in response to vibrating muscle. Seroussi et al. [118] evoked acute vertical sinusoidal vibrations $(f=3-10 \mathrm{~Hz}$; $A=0.4-13 \mathrm{~mm}$ ) from a servohydraulic shaker, and were successful in removing motion artefacts by passing the raw EMG through a phaseless digital 6 pole Butterworth high pass filter with a cutoff frequency of $30 \mathrm{~Hz}$. They found that when EMG of the erector spinae was adjusted for torque, a significant increase (19\%) in mean torque was observed. Likewise, Bongiovanni and Hagbarth 
[11] reported that tibialis anterior EMG activity and single motor unit discharge were augmented from a pneumatic vibratory unit $(f=150 \mathrm{~Hz}, A=1.5 \mathrm{~mm})$.

Using a small actuator $(f=50 \mathrm{~Hz}, A=5 \mathrm{~mm})$ applied acutely to the quadriceps, Warman et al. [132] reported an increase in $\mathrm{EMG}_{\mathrm{rms}}$ of the rectus femoris during isometric (30\%), isokinetic (43\%), and concentric (107\%) contractions. In a subsequent study, Humphries et al. [58] used the same vibrating actuator and reported no significant differences in peak normalised $\mathrm{EMG}_{\mathrm{rms}}$ between acute vibration and resting conditions. Additionally, there were no corresponding changes in the rate of force development or peak force. However, the vibration exposure duration was not documented, therefore it may have been too short or too long to elicit the desired responses. Similarly, Torvinen, et al. [126] found no significant changes in soleus mean power frequency and $\mathrm{EMG}_{\mathrm{rms}}$ from $4 \mathrm{~min}$ of $\mathrm{VbX}(f=25-40 \mathrm{~Hz}, A=2 \mathrm{~mm}$, VV) ( $\odot$ Table 2), but reported a decrease in mean power frequency and $\mathrm{EMG}_{\mathrm{rms}}$ for the vastus lateralis and gluteus medius muscles, which was accompanied by no changes in muscle function.

In a subsequent study, Torvinen et al. [123] used the same exercise routine and time constructs as the previous study, but in the latter study the participants performed the exercise routine on a SV platform and the vibration frequency was incrementally increased from $15-30 \mathrm{~Hz}(A=10 \mathrm{~mm})$. During $\mathrm{VbX} \mathrm{EMG}_{\mathrm{rms}}$ was significantly augmented in the soleus and gastrocnemius but there was no change in vastus lateralis $\mathrm{EMG}_{\mathrm{rms}}$. However, an increase in isometric leg force and $\mathrm{VJ}$ height was reported. The authors provided no discussion on the dissonance between the 2 studies, consequently the reader can only surmise, that either the type of vibration platform (VV vs. SV) or vibration parameters ( $f=15-30$ vs. $25-45 \mathrm{~Hz}, A=2 \mathrm{~mm}$ vs. $10 \mathrm{~mm}$ ) may explain the variation in results. However, it is feasible that the action of the side alternating platform with a larger amplitude may have contributed to the increase in muscle performance. Recent evidence has reported that using a fixed frequency $(30 \mathrm{~Hz})$ and amplitude $(4 \mathrm{~mm})$, a SV platform generates greater muscle activation of lower limb muscles compared to a VV platform [1].

\section{Upper body}

The effect of acute upper-body VbX on EMG activity has produced similar results to that of acute lower-body vibration. Using a vibrating dumbbell, Bosco et al. [12] observed that during acute intermittent vibration $(f=30 \mathrm{~Hz})$ biceps brachii $\mathrm{EMG}_{\mathrm{rms}}$ increased 2-fold compared to baseline measurements, and during post-vibration bicep power was augmented but no corresponding increase in EMG activity was found. Further evidence of increased $\mathrm{EMG}_{\mathrm{rms}}$ has been observed from vibrating isometric elbow pull and push actions, with increases in co-contraction at loads of $20 \%$ and $40 \%$ of maximum force [84]. The authors speculate that the mechanism for increased $\mathrm{EMG}_{\mathrm{rms}}$ cannot be entirely accounted for by spinal reflexes but by increased motor unit synchronisation and firing frequency. Although Mischi and Cardinale's [84] study provides some new insights to the response of superimposing acute vibration on agonist, antagonist and co-contraction of muscles, caution is required because only one vibration frequency $(28 \mathrm{~Hz})$ was tested with brief exposures of vibration and the recorded absence of amplitude restricts the findings of the study. Conversely, Moran et al. [85] reported that when a custom built vibrating unit was directly placed on the bicep brachii tendon $(f=65 \mathrm{~Hz}, A=1.2 \mathrm{~mm})$ it did not elicit an increase in $\mathrm{EMG}_{\mathrm{rms}}$ during the lifting phase of bicep curls at 70\% 1RM. Similarly, post-vibration showed no enhancement in $\mathrm{EMG}_{\mathrm{mpf}}$ (mean power frequency) and peak force or power.

Finally, a novel study was conducted to investigate acute muscle activity on a VV platform on both the lower (vastus lateralis, and biceps femoris) and upper limb muscles (biceps brachii and triceps brachii) [54]. The investigators used a range of frequencies $(f=25,30,35,40,45 \mathrm{~Hz})$, amplitudes $(A=2$ and $4 \mathrm{~mm})$, and body positions of static squat, dynamic squat, static bicep curl and dynamic bicep curl. They reported that a static squat $\mathrm{EMG}_{\mathrm{rms}}$ of vastus lateralis and biceps femoris was augmented when vibration frequency and amplitude were increased, but no increase in muscle activity was evident in static and dynamic bicep curls, probably because the transmission of vibration was damped by the lower extremity. It is important to note that the raw EMG signal was passed through a sixth order Butterworth filter between $100 \mathrm{~Hz}$ and $450 \mathrm{~Hz}$, which may have removed important muscle activity signals.

\section{Posture}

Roelants et al. [107] has recently determined whether posture affects muscle activation by investigating 3 different isometric squat positions of a two-leg, high squat (knee angle $125^{\circ}$, hip angle $140^{\circ}$ ); two-leg, low squat (knee and hip angle $90^{\circ}$ ); and one-leg, high squat (knee angle $125^{\circ}$, hip angle $140^{\circ}$ ). The investigators reported that acute $\mathrm{VbX}$ increased $\mathrm{EMG}_{\mathrm{rms}}$ activity more in a one-leg high squat, compared to two-leg high and low squats. However, further testing is required to determine if any EMG differences exist in squatting positions that occur between $90^{\circ}$ and $125^{\circ}$ knee flexion.

Abercromby et al. [1] acutely investigated muscle activity of different lower limb postures of static squat $\left(18.5^{\circ}\right)$ and dynamic squat (eccentric and concentric $10-35^{\circ}$ knee angle) performed on both SV and VV platforms ( $f=30 \mathrm{~Hz}, A=4 \mathrm{~mm}$ ). For isometric, eccentric and concentric muscle action, $\mathrm{EMG}_{\mathrm{rms}}$ increased significantly in all 4 lower limb muscles for both SV and VV. In support of this, Delecluse et al. [43] reported that standing in a static half-squat position on a VV platform $(f=35 \mathrm{~Hz}, A=5 \mathrm{~mm}, a=3.9 \mathrm{~g})$ increased gastrocnemius and rectus femoris $\mathrm{EMG}_{\mathrm{rms}}$ activity, compared to placebo $(a=0.4 g$ ) control (no vibration); however, the sampling period was only conducted over 20 s. Conversely, Cormie et al. [36] observed that in a half-squat position (knee angle $100^{\circ}$ ) there were no changes in average iEMG (integrated) activity of the vastus lateralis, vastus medialis, bicep femoris during acute vibration treatment $(f=30 \mathrm{~Hz}, A=2.5 \mathrm{~mm}$, VV [Power Plate]).

\section{The effects of vibration exercise on cardiovascular indices \\ Blood flow}

Hand-held tools are normally operated at high vibration (80$100 \mathrm{~Hz}$ ), which has been shown to decrease blood flow to the digits of the hand, resulting in 'white finger vibration' [20]. However, Kerschan-Schindl et al. [67] were first to report that acute $\operatorname{VbX}(f=26 \mathrm{~Hz}, A=3 \mathrm{~mm}$, SV [Galileo] $)$ increased blood flow of the popliteal artery $(100 \%)$ and caused erythema in the foot and calf. Furthermore, Lythgo et al. [76] found that when an acute intermittent vibration protocol was performed $(f=10 \mathrm{~Hz}-30 \mathrm{~Hz}$, SV [Galileo]), an increase in mean blood cell velocity of the femoral artery was evident, with $30 \mathrm{~Hz}$ providing the greatest increase in blood flow compared to resting levels. In contrast, Hazell et al. [55] found that an acute intermittent vibration 
protocol ( $f=45 \mathrm{~Hz}, A=2 \mathrm{~mm}$, VV [Wave]) reported no increases in femoral artery blood flow after 3 min. Likewise, Button et al. [20] reported no significant differences in leg blood flow of a vibrating cushion ( $f=60 \mathrm{~Hz}, A=$ not given, VV [ATL]) placed under the gluteal muscles, and a hand unit placed under the right foot while in the seated position. In this particular study, no rationale was given for using a hand-held device applied to the foot, or why the vibrating cushions were acutely applied to the gluteals rather than directly to the legs. Additionally, the participants were seated with knees and ankles at $90^{\circ}$, which suggests that vibration transmission may have been damped, attenuating blood flow.

Vibration massage has also been shown to increase skin blood flow. 3 min of acute continuous vibration $(f=30 \mathrm{~Hz}, A=5-6 \mathrm{~mm}$, VV [Power Plate]) increased gastrocnemius skin blood flow by $250 \%$ compared to baseline, but there were no treatment differences between with and without vibration [72]. Moreover, Maloney-Hinds et al. [78] found that when the forearm was passively vibrated on a VV platform [Power Plate] at $30 \& 50 \mathrm{~Hz}$ $(A=5-6 \mathrm{~mm})$ for $10 \mathrm{~min}$ it significantly increased skin blood flow within $5 \mathrm{~min}$ and remained elevated for $9 \mathrm{~min}$ post-vibration.

\section{Heart rate, blood pressure and arterial stiffness}

Acute VbX has been reported to have little effect on heart rate (HR) and blood pressure $[20,55,90]$. Kerschan-Schindl et al. [67] observed no change in HR, systolic and diastolic blood pressure values after acute $\mathrm{VbX}(f=26 \mathrm{~Hz}, A=3 \mathrm{~mm}$, SV [Galileo]). However, exhaustive VbX did increase HR and systolic pressures by $30 \%$ and $15 \%$ [ 101$]$. To date, only one study has investigated the effects of vibration on arterial stiffness by measuring brachialankle pulse velocity [90]. Following acute intermittent vibration $(f=26 \mathrm{~Hz}, A=2-4 \mathrm{~mm}, \mathrm{VV}$ [Power Plate]) arterial stiffness was significantly reduced (3\%) at 20 and 40 min post-vibration compared to the same time series of no vibration.

\section{The effects of vibration exercise on muscle function} Power

\section{Acute vibration - lower body power}

A summary of the studies that examined the acute effects of VbX on lower and upper body power can be found in 0 Table 2, 3. Vertical countermovement jump (CMJ) has been used by many investigators to assess muscle power and its acute effects on $\mathrm{VbX}$. The enhancement in $\mathrm{VJ}$ height has varied across the different research protocols. For instance, using a SV (Galileo) platform, Cochrane and Stannard [32] reported an $8.2 \%$ increase in $\mathrm{CMJ}$ height from 5 continuous minutes of acute vibration $(f=26 \mathrm{~Hz}, \quad A=6 \mathrm{~mm})$ (० Table 2), and Torvinen et al. [123] observed a $2.5 \%$ enhancement following 4 continuous minutes of vibration ( $f=15-30 \mathrm{~Hz}, A=10 \mathrm{~mm}$ ). Likewise, $5 \mathrm{~min}$ of continuous $\mathrm{VbX}$ has been shown to raise muscle temperature by $1.5^{\circ} \mathrm{C}$, which significantly increased CMJ height (9.3\%) and power ( $4.4 \%$ ) but when the muscle temperature was elevated by the same amount in stationary cycling and hot water bath the increases in CMJ were similar between the 3 conditions [34].

Studies that have used VV platforms (Power Plate, Nemes) have shown similar findings. Bosco et al. [15] has reported an increase in VJ height of $4 \%$ from 10 min of intermittent vibration $(f=26 \mathrm{~Hz}$, $A=4 \mathrm{~mm}$, VV [Nemes]). Brief, single vibration ( $f=30 \& 40 \mathrm{~Hz}$, $A=2-4 \mathrm{~mm}$ ) exposures of $30 \mathrm{~s}$ and $45 \mathrm{~s}$ performed on a VV platform have respectively recorded a $0.6 \%$ and $9 \%$ improvement in VJ height [8,36]. Conversely, Torvinen et al. [126] found no significant increases in VJ height when dynamic exercise was combined with $4 \mathrm{~min}$ of $\mathrm{VbX}(f=25-40 \mathrm{~Hz}, A=2 \mathrm{~mm}$, VV [Kuntatory]). It is difficult to explain why Torvinen et al.'s [123] earlier study reported a $4.4 \%$ increase, as both studies used similar protocols and participants. However, a VV platform was used in the latter study where the amplitude was smaller $(2 \mathrm{~mm})$ than the previous study $(10 \mathrm{~mm})$, which could have negated the desired responses. The use of $\mathrm{VbX}$ to cause acute changes in power would appear similar to using contrast-complex training, a modality also believed to utilise the theory of post-activation potentiation PAP), which is referred to as an increase in muscle performance preceded by a muscle contractile activity [111] that involves myogenic and neurogenic factors [56]. To date, it is equivocal whether acute VbX causes PAP by enhancing muscle twitch and reflex properties [33,35,40,64, 79, 92].

Measuring muscle power has not only been confined to vertical jump. Leg press, weighted squats, and knee extension have also been used to assess muscle power from an acute bout of $\mathrm{VbX}$. Bosco et al. [14] found a 6-8\% increase in single leg press power across loads of $70,90,100,139 \mathrm{~kg}$ in elite volleyball players $(\mathrm{n}=6)$ from intermittent $\mathrm{VbX}(f=26 \mathrm{~Hz}, A=10 \mathrm{~mm}$, SV [Galileo 2000]). Similarly, Rhea and Kenn [98] observed a 5.2\% increase in squat power ( 3 reps, $75 \% 1 \mathrm{RM}$ ) of male college athletes that received vibration $(f=35 \mathrm{~Hz}, A=4 \mathrm{~mm}$, VV) while dynamic squatting their body weight. However, when ballistic knee extensions were performed with direct vibration $(f=65 \mathrm{~Hz}, A=1.2 \mathrm{~mm})$ that was applied to distal tendon of quadriceps no significant changes in power variables were found [75].

\section{Acute vibration - Upper body power}

The effects of acute vibration on upper-body power have been explored less so, and with varying results. Bosco et al. [12] found a post-vibration $8 \%$ increase in average power of bicep brachii from 12 international boxers that isometrically gripped a handheld vibrating device $(f=30 \mathrm{~Hz}, A=6 \mathrm{~mm})$. In a similar study, Issurin and Tenenbaum [60] used an isotonic vibrating cable ( $f=44 \mathrm{~Hz}, A=3 \mathrm{~mm}$ ) where non-elite and elite athlete performed 3 sets of 3 reps (65-70\% $1 \mathrm{RM})$ at a tempo of $2 \mathrm{~s}$ per rep. They reported that both non-elite and elite athlete groups produced a $10.2 \%$ and $10.7 \%$ increase in mean power and $10.4 \%$ and $7.9 \%$ increase in peak power during vibration compared to no vibration.

Further support of vibration enhancing upper-body power has been reported by Poston et al. [94], where an Olympic barbell was fitted with a vibrating electric motor and experienced weight lifters performed an isometric vibration bench press hold $(f=30 \mathrm{~Hz}, A=1.1 \mathrm{~mm})$ for $30 \mathrm{~s}$ between the second and third sets of bench pressing ( 3 reps, 70\% 1RM). Although, the average power of the bench press was higher with vibration than without, there were discrepancies in baseline power outputs, suggesting that either the athletes should have been blinded before receiving vibration or a greater familiarisation period with the vibration apparatus should have been included.

Some researchers have shown little or no effect of vibration on upper-body power. Moran et al. [85] observed no significant prepost changes in power, moment, and angular velocity when a custom built vibratory unit $(f=65 \mathrm{~Hz}, A=1.2 \mathrm{~mm})$ was directly applied to the tendon of bicep brachii while resistance trained males performed 3 sets $x 5$ reps of bicep curls (70\% 1RM). The same group of researchers [74] repeated a similar experiment and used the same vibratory unit ( $f=65 \mathrm{~Hz}, A=1.2 \mathrm{~mm}$ ), and participants but with the addition of $40 \%$ and $70 \% 1 \mathrm{RM}$ loading with vibration did not produce any significant changes in power 


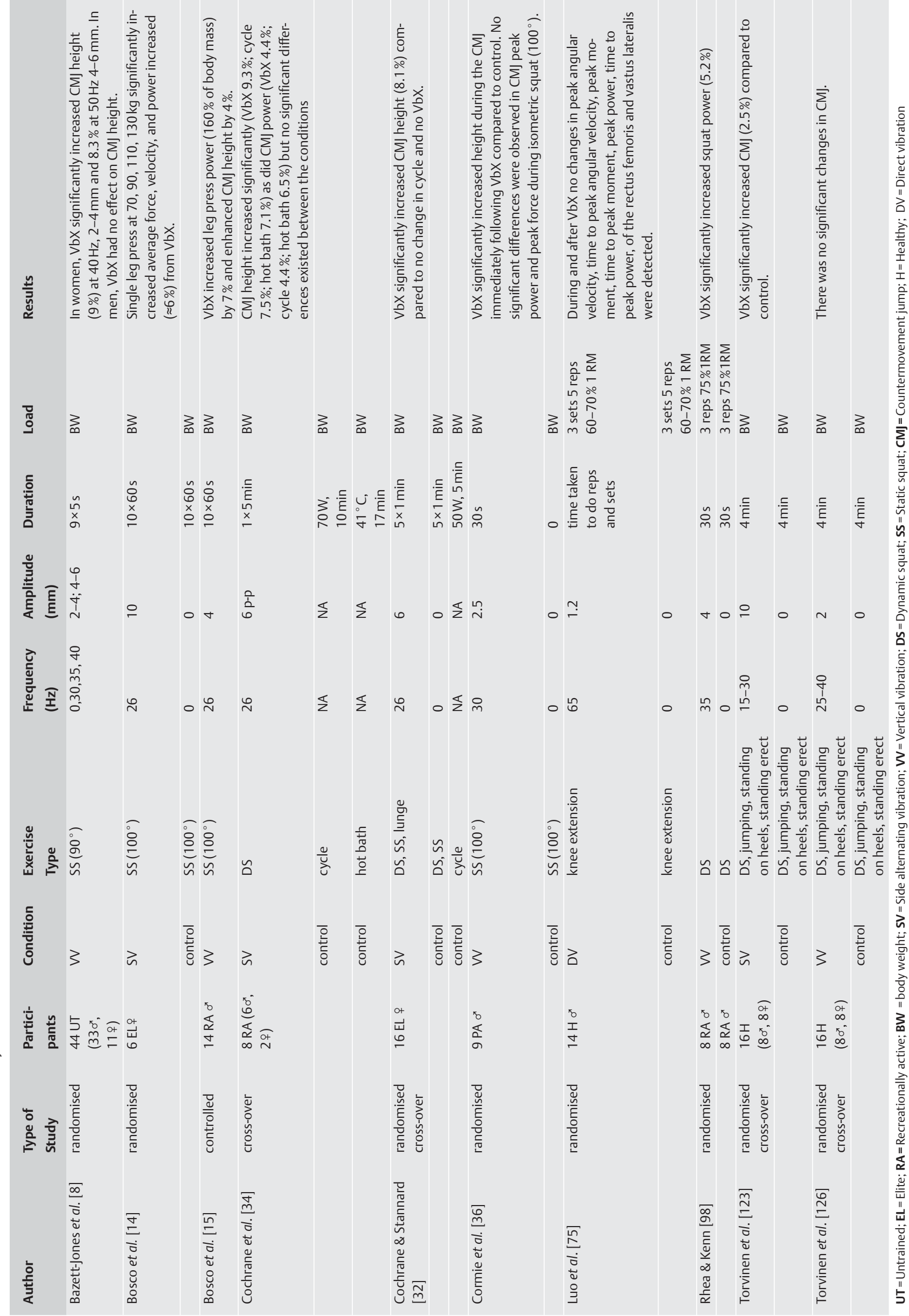



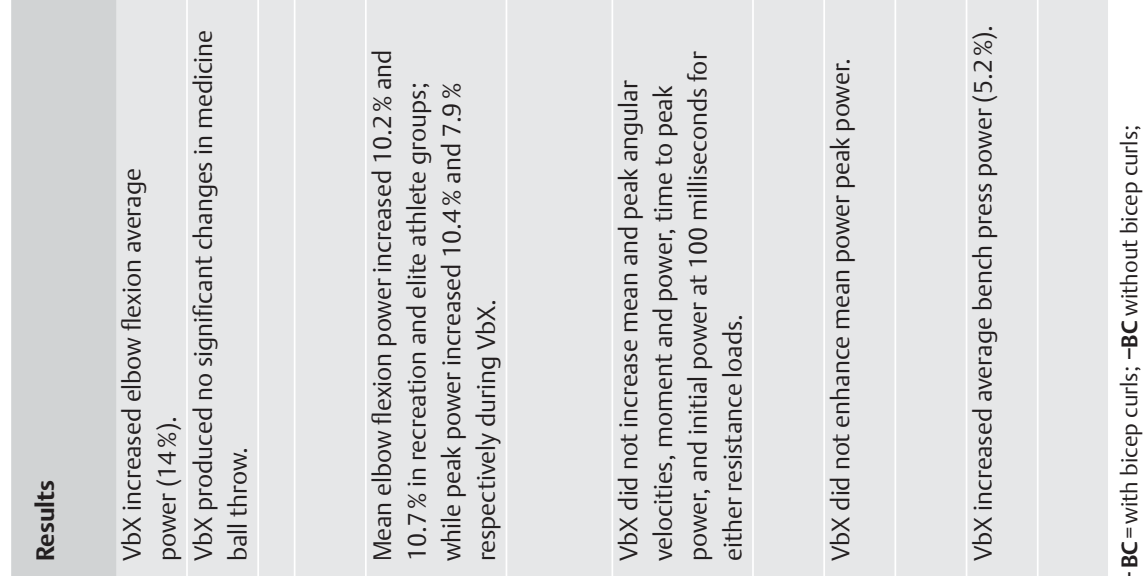

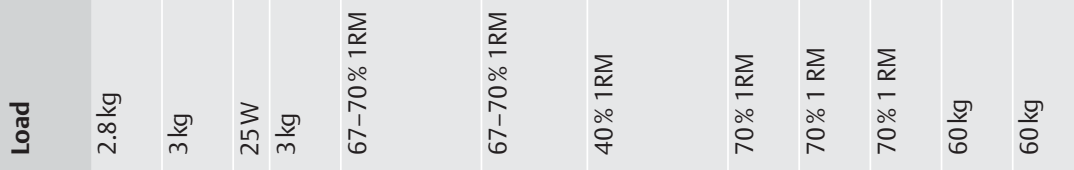

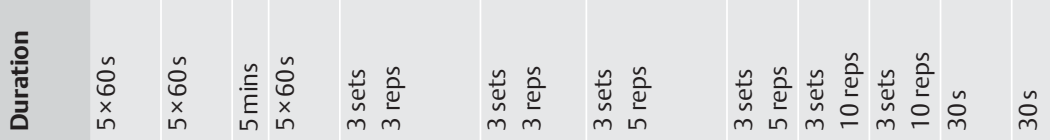

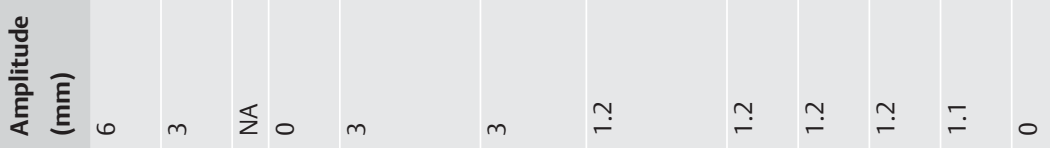

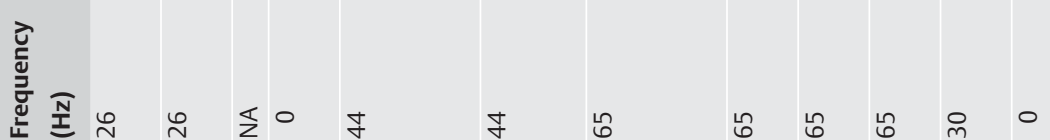

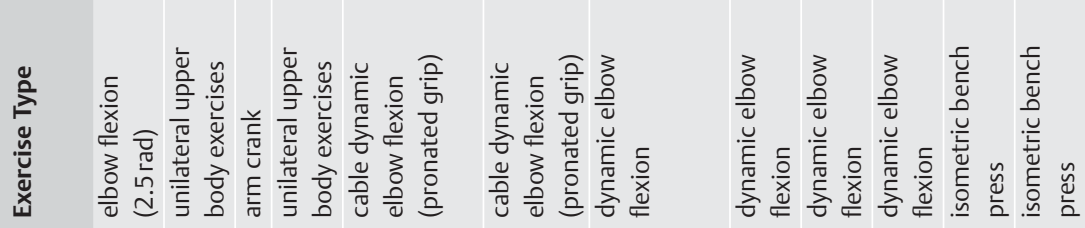

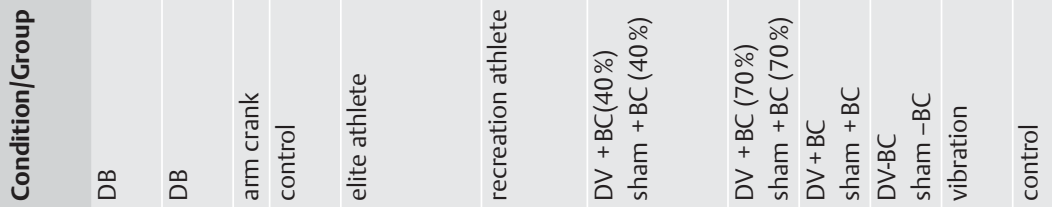

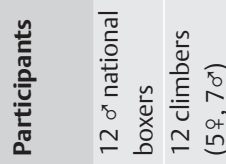

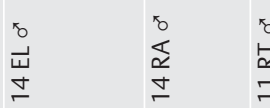

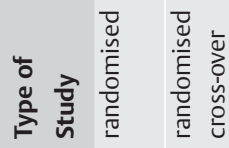

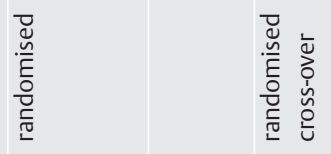

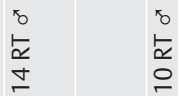


measures. From these results the researchers proposed that when performing dynamic exercise, such as bicep curls, it relies on the stretch-shortening cycle where smaller vibration amplitude may be required to activate and optimise muscle spindle sensitivity. However, Bosco et al.'s [12] findings contradict this because they reported an $8 \%$ increase in bicep brachii peak power from $5 \mathrm{~min}$ of intermittent dumbbell vibration $(f=30 \mathrm{~Hz}$, $A=6 \mathrm{~mm}$ ). Therefore, the direct application of the vibratory unit and its frequency $(65 \mathrm{~Hz})$ may have caused the insignificant findings from Moran et al. [85] and Luo et al. [74]. Moreover, Cochrane and Hawke [29] have also reported no significant increases in upper-body power of climbers that were exposed to an electric powered vibrating dumbbell $(3 \mathrm{~kg})(f=26 \mathrm{~Hz}, A=3 \mathrm{~mm}$, [TOP Galileo]). At present the research has focussed on shortand long-term lower body power but there is lack of conclusive research on longer exposures of $\mathrm{VbX}$ on upper body power.

\section{Short-term vibration ( $\leq 2$ months) - Lower body power} Repeated single bouts of vibration performed over days or weeks have been investigated and the findings of the short-term $\mathrm{VbX}$ on lower body and upper body power can be found in $\odot$ Table 4, 5 . Bosco et al. [13] reported a 11.9\%. increase in VJ height ( 5 s continuous jumping protocol) in handball and waterpolo players after receiving 10 days of intermittent $\mathrm{VbX}(f=26 \mathrm{~Hz}, A=10 \mathrm{~mm}$, $\mathrm{SV}$, [Galileo]). Further support for short-term vibration potentiating CMJ has been reported by Fagnani et al. [49] where competitive female athletes increased their $\mathrm{CMJ}$ by $8.7 \%$ from 8 weeks of vibration training $(f=35 \mathrm{~Hz}, A=4 \mathrm{~mm}$, VV [Nemes]). Well-trained strength males (21-40yrs) that underwent 5 weeks ( $3 \mathrm{x} /$ week) of vibration $(f=40 \mathrm{~Hz}, A=$ not given, $\mathrm{VV}$ [Nemes]) combined with 6RM squats reported an increase in VJ by $8.8 \%$ [108]. Annino et al. [4] observed an increase in VJ height (6.3\%) in welltrained ballerinas after performing 2 months (3x/week) of $\mathrm{VbX}$ $(f=30 \mathrm{~Hz}, A=5 \mathrm{~mm}, \mathrm{VV}$ [Nemes]) additionally, the average power, force and velocity of leg press increased significantly at loads of 50,70 , and $100 \mathrm{~kg}$. In a recent study [44] where vibration frequency was set individually for each participant, the authors reported that after 8 weeks (3x/week) of vibration training, squat jump height increased by $11 \%$, continuous rebound jump height was enhanced by $22 \%$ and mean power significantly increased by $18 \%$ respectively compared to a fixed $(30 \mathrm{~Hz})$, or no vibration . But no significant increases in $\mathrm{CMJ}$ height were found after 8 weeks in all 3 groups. Likewise, other vibration training protocols conducted over different time periods such as, 9 days [30], and 5 weeks, (3x/week) [42] have reported no significant increases in VJ.

\section{Long-term vibration (> 2 months) - Lower body power}

Several studies have investigated the long-term effects of vibration on muscle power using VJ performance. The majority of these studies have found increases in VJ jump performance. Delecluse et al. [43] randomised 67 untrained females (21 yrs) into VbX $(f=35-40 \mathrm{~Hz}, A=2.5-5 \mathrm{~mm}$, VV [Power Plate]), resistance training (8-20RM of knee extension and leg press), placebo and control groups and found that after 12 weeks (3x/week) of training, VJ height increased by $7.6 \%$ from VbX. Likewise, healthy non-athletic males and females increased VJ height by $9.0 \%$ and $7.7 \%$ following 4 and 8 months of $\mathrm{VbX}(f=25-40 \mathrm{~Hz}, A=2 \mathrm{~mm}$, VV [Kuntotary]) [124-125]. In addition, post-menopausal women have shown increases in VJ height of $19.4 \%$ and $4.7 \%$ from 24 weeks (3x/week) [106] and 6 months (2x/week) of VbX training [110]. However, no significant improvements in VJ height have been reported from 11 weeks (3x/week) [41] of $\mathrm{VbX}$.
In summary, there is strong evidence that acute $\mathrm{VbX}$ can enhance upper and lower-body muscle power, and there is some indication that VbX can enhance lower and upper-body muscle power over a longer-term, although this is less convincing. Future studies need to be conducted to determine the optimal duration of the rest between repetitions, the optimal frequency and duration necessary to maximise power in both short and long term studies.

Force

Acute vibration - Upper body force. There are many methods of applying vibration to the upper-body; some researchers have used custom-built vibratory units applied directly to the muscle or attached to resistant training cables, while in recent times commercially manufactured vibrating dumbbells have also become available. A summary of the studies that investigated the acute effects of vibration on upper and lower body force can be found in 0 Table 6, 7. In a study conducted by Kin-Isler and colleagues [68], an electromotor was used to transmit vibrations through a cable attached to a leather belt that was placed over the belly of the biceps brachii. Using a range of vibration frequencies $(f=6,12,24 \mathrm{~Hz}, A=4 \mathrm{~mm})$ and joint angles $\left(90^{\circ}, 120^{\circ}\right.$, $\left.150^{\circ}\right)$ the researchers reported that during a $10 \mathrm{~s}$ vibration exposure, a $6.4 \%$ increase in MVC elbow flexors was observed. However, the length of muscle (conducted at various angles) did not affect isometric MVC. Finally, vibration has no effect on grip force when using an electric powered vibrating dumbbell [29] or when arms are exposed less proportionally to the legs on a vibrating platform $[32,123,126]$.

\section{Acute vibration - Lower body force}

There is little consensus on whether acute vibration increases lower-body force of isometric, isokinetic and isoinertial muscle actions. De Ruiter et al. [58] reported that when the knee extensors were electrically stimulated, the maximum force-generating capacity and isometric contraction significantly declined after an intermittent acute bout of vibration $(f=30 \mathrm{~Hz}, A=8 \mathrm{~mm}$, SV, [Galileo 2000]). However, no changes in maximal isometric leg extensor strength were found following $4 \mathrm{~min}$ of vibration ( $f=25-40 \mathrm{~Hz}, A=2 \mathrm{~mm}, \mathrm{VV}$ [Kuntotary]; [126]. Conversely, in a follow-up experiment using the same design and experimental protocol, the researchers [123] reported an increase of $3.2 \%$ in leg extensor strength. The use of different vibration machines and protocols could explain the discrepancy in results. It is possible that the SV elicited a greater response in leg extensor strength compared to the VV machine; this has recently been confirmed by Abercromby et al. [2]. However, other studies from Humphries et al. [58], Kemertzis et al. [66], and Erskine et al. [48] have indicated that acute vibration does not enhance isometric or isokinetic force and claim that inhibition rather than facilitation occurs. On the contrary, there have been reports that vibration does increase force attributes with Warman et al. [132] reporting that direct vibration increased isotonic knee extensor force, but no changes were seen in isometric or isokinetic force ( Table 7). However, using a vibration platform of 2 and $6 \mathrm{~min}$ exposure $(f=26 \mathrm{~Hz})$, Stewart et al. [120], and Jacobs and Burns [63] have reported increases in knee isokinetic peak torque of $3.8 \%$ and $7.7 \%$ respectively and Mileva et al. [83] reported an increase in isoinertial peak torque by $25 \%$ and $12 \%$ using loads of $30 \%$ and $70 \% 1 \mathrm{RM}$ knee extension with a vibrating system $(f=10 \mathrm{~Hz}, A=$ not given $)$. 

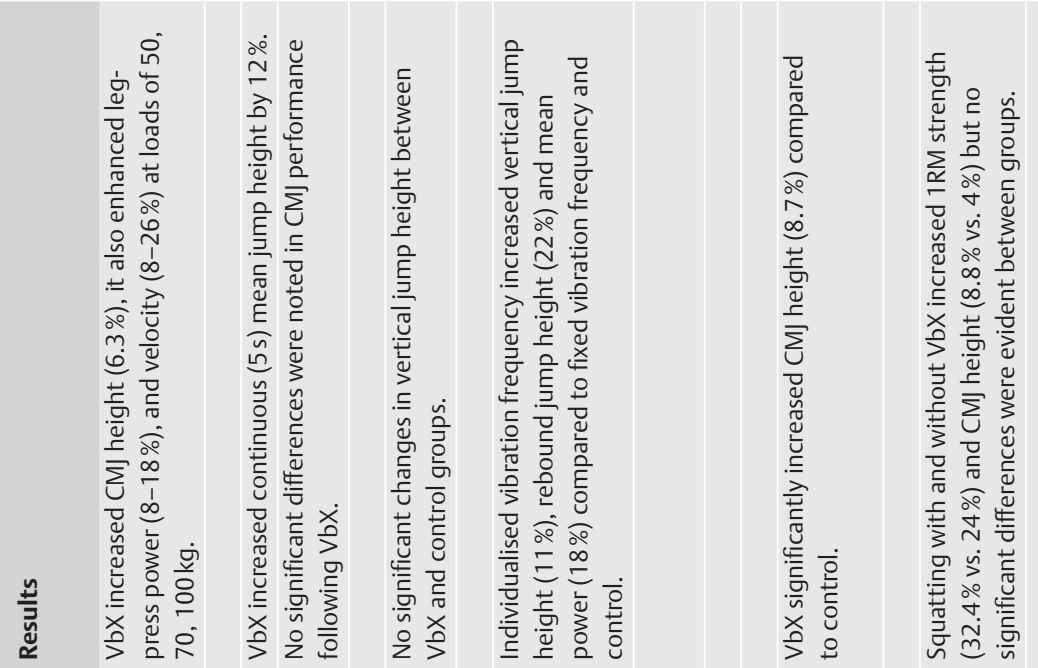

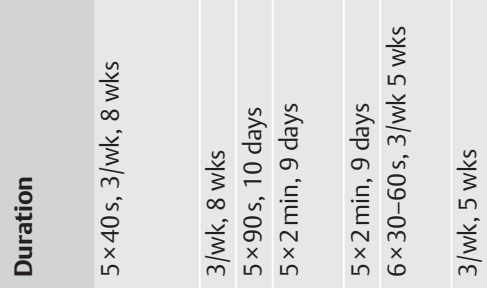

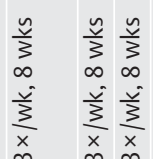

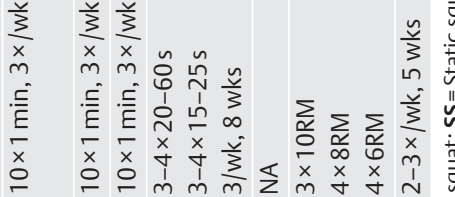

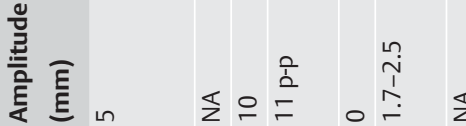

n n t $\frac{\pi}{2} \frac{\alpha}{2}$

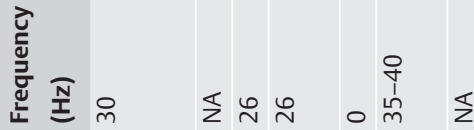

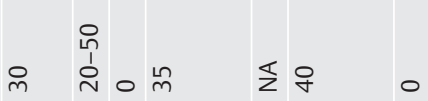

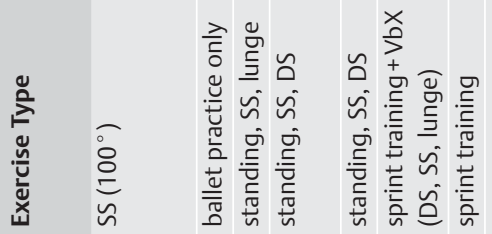

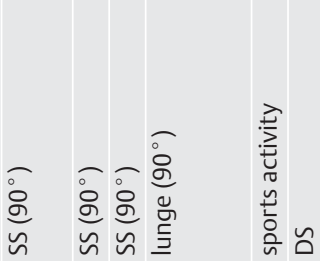

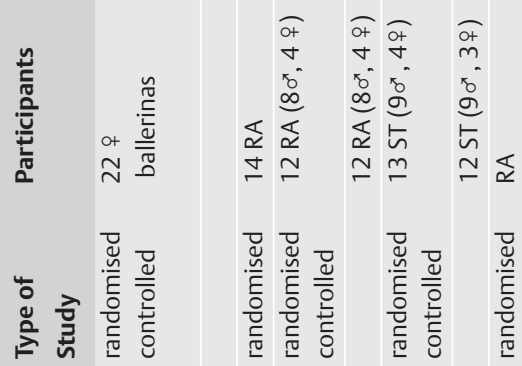

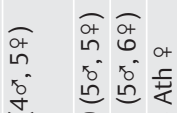

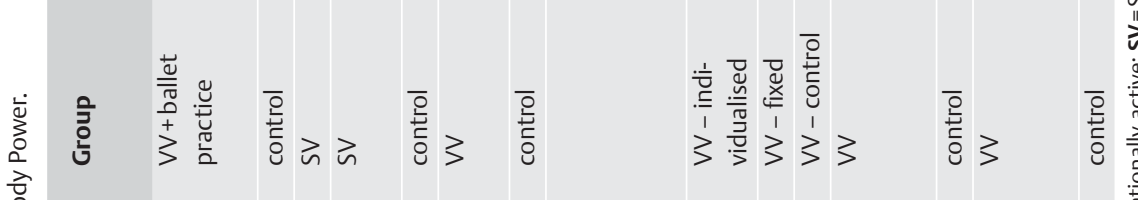

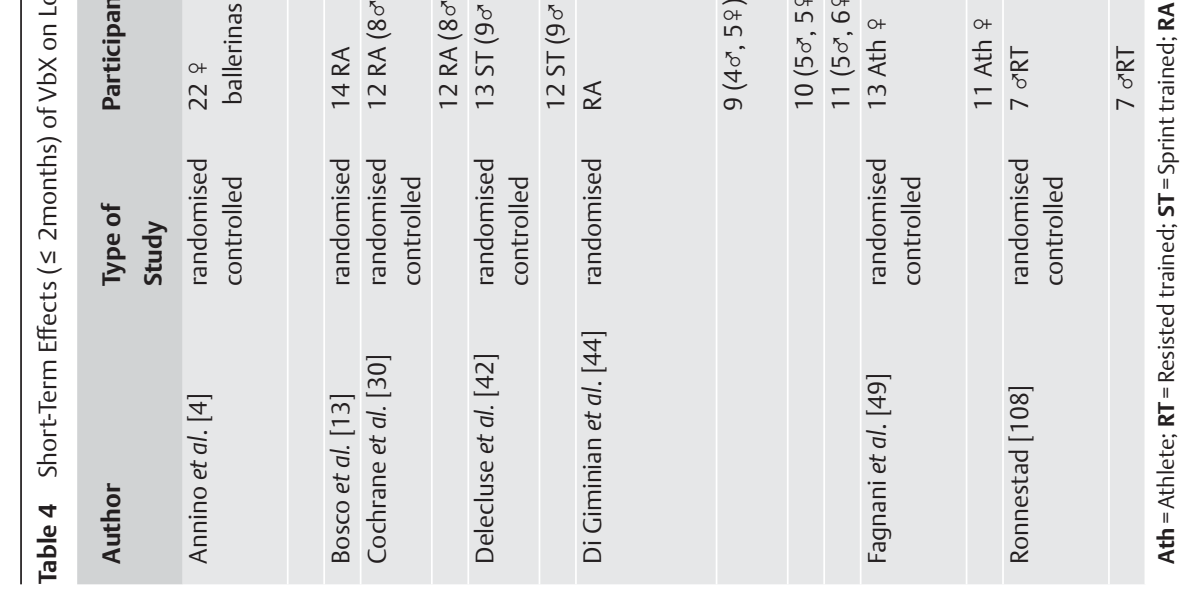



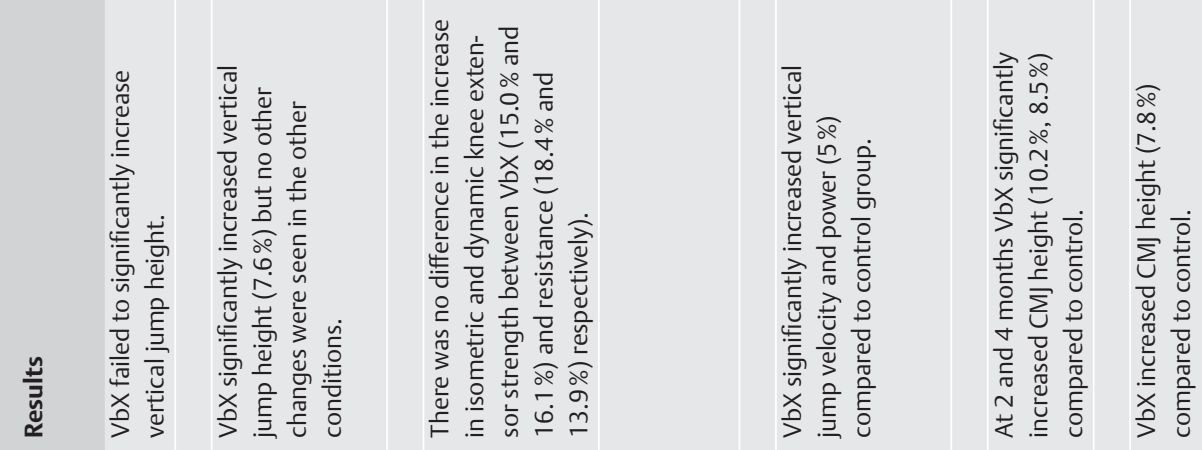

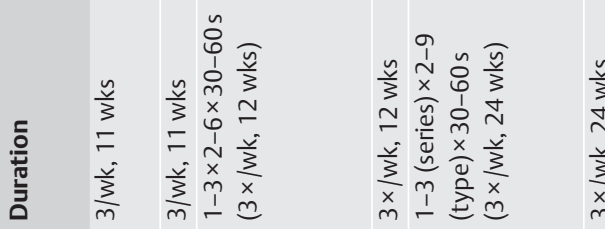
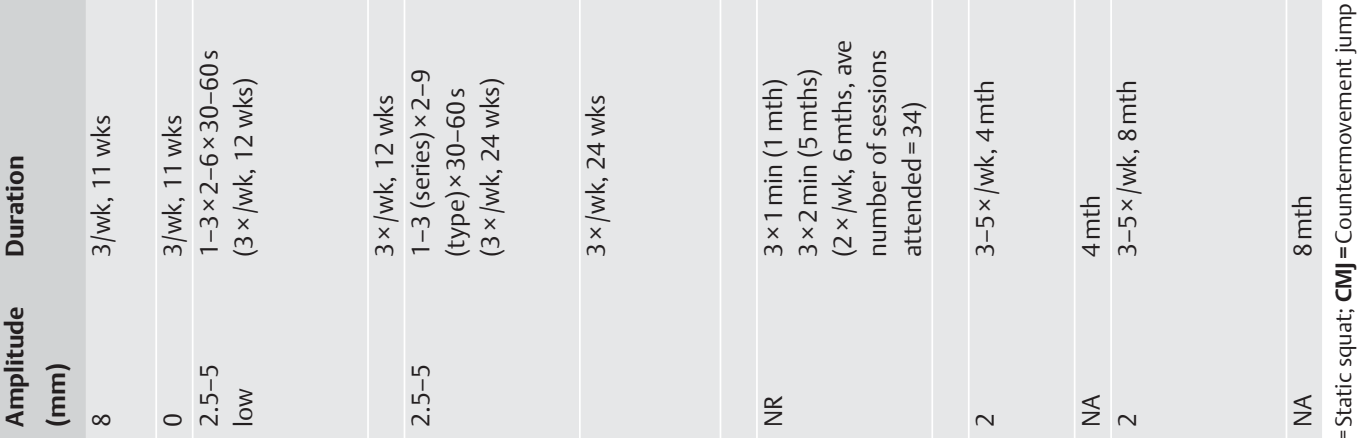

$\frac{a}{z}$

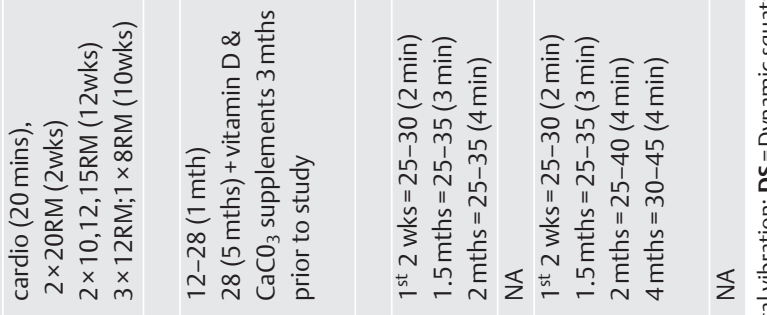

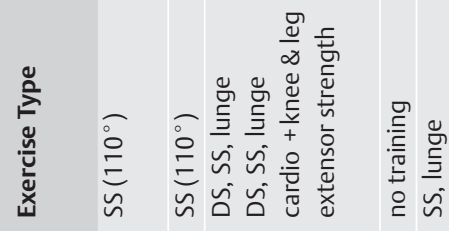
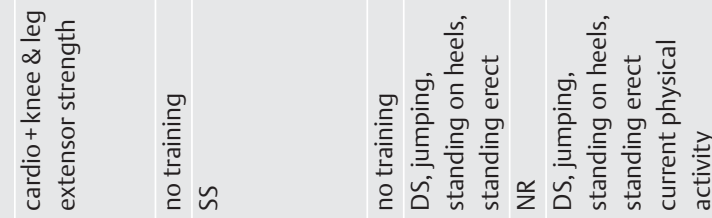

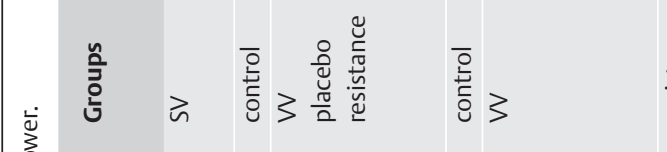

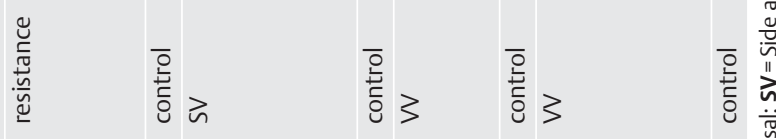

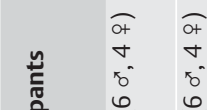

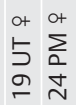

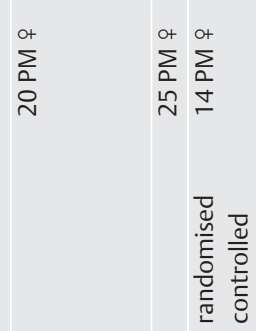

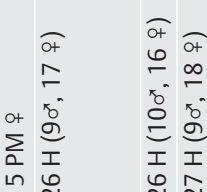
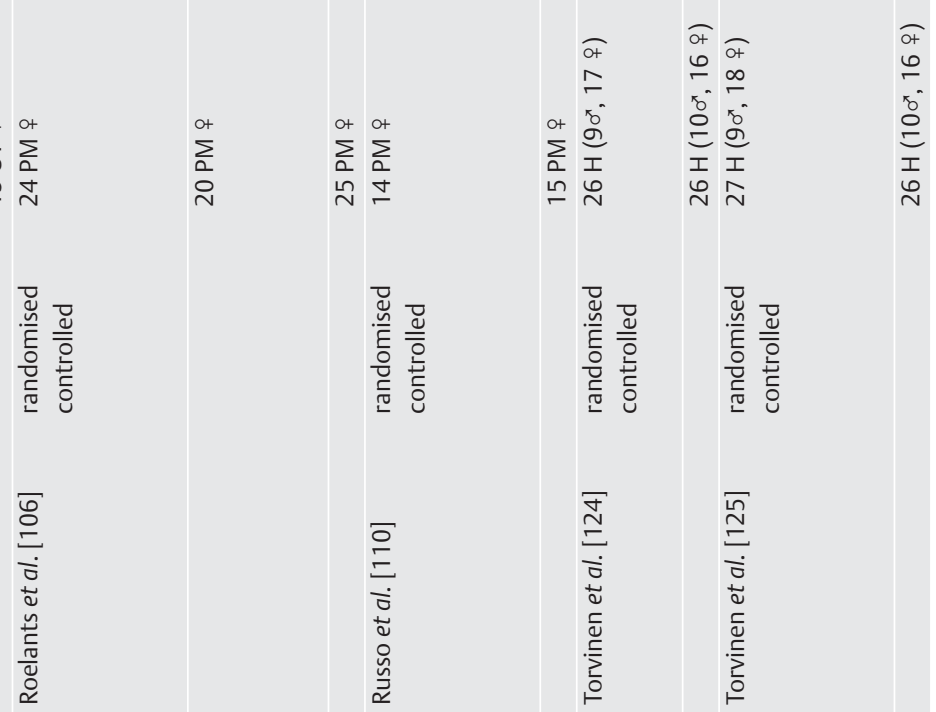


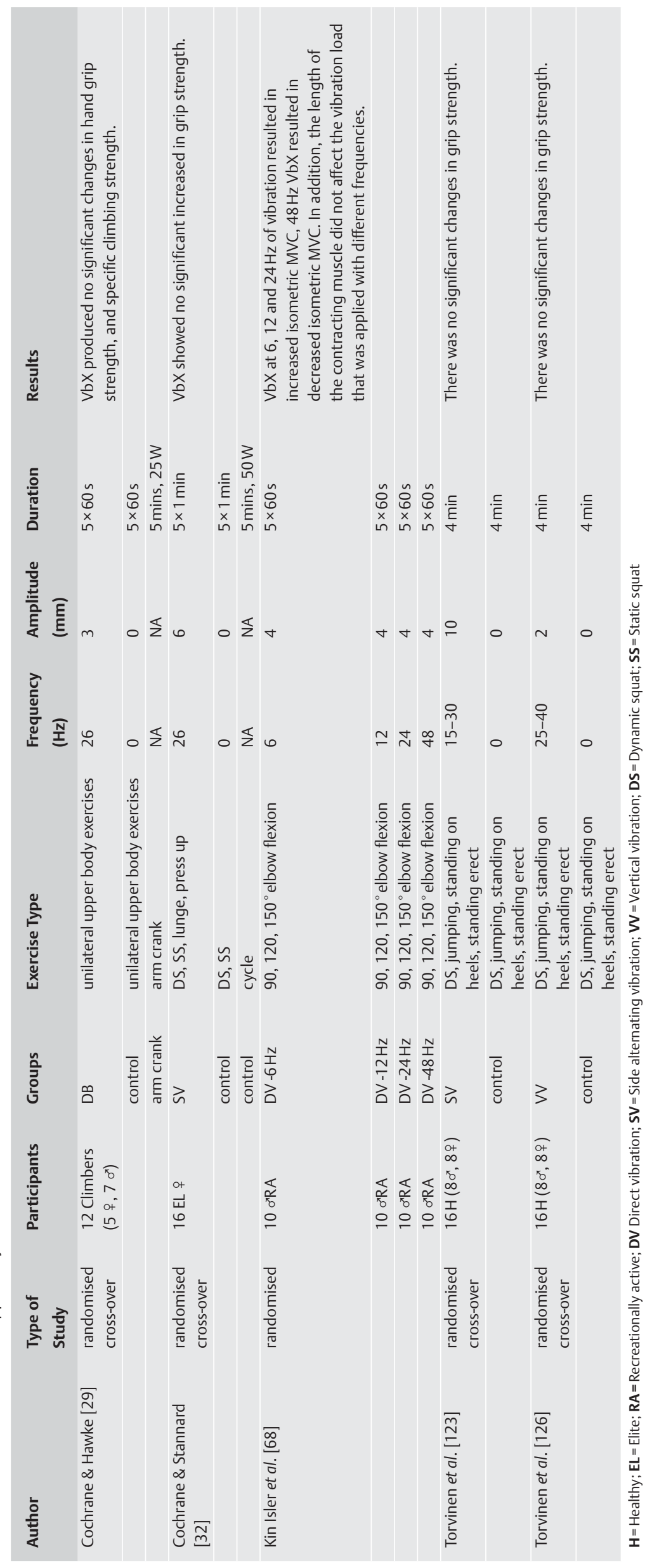




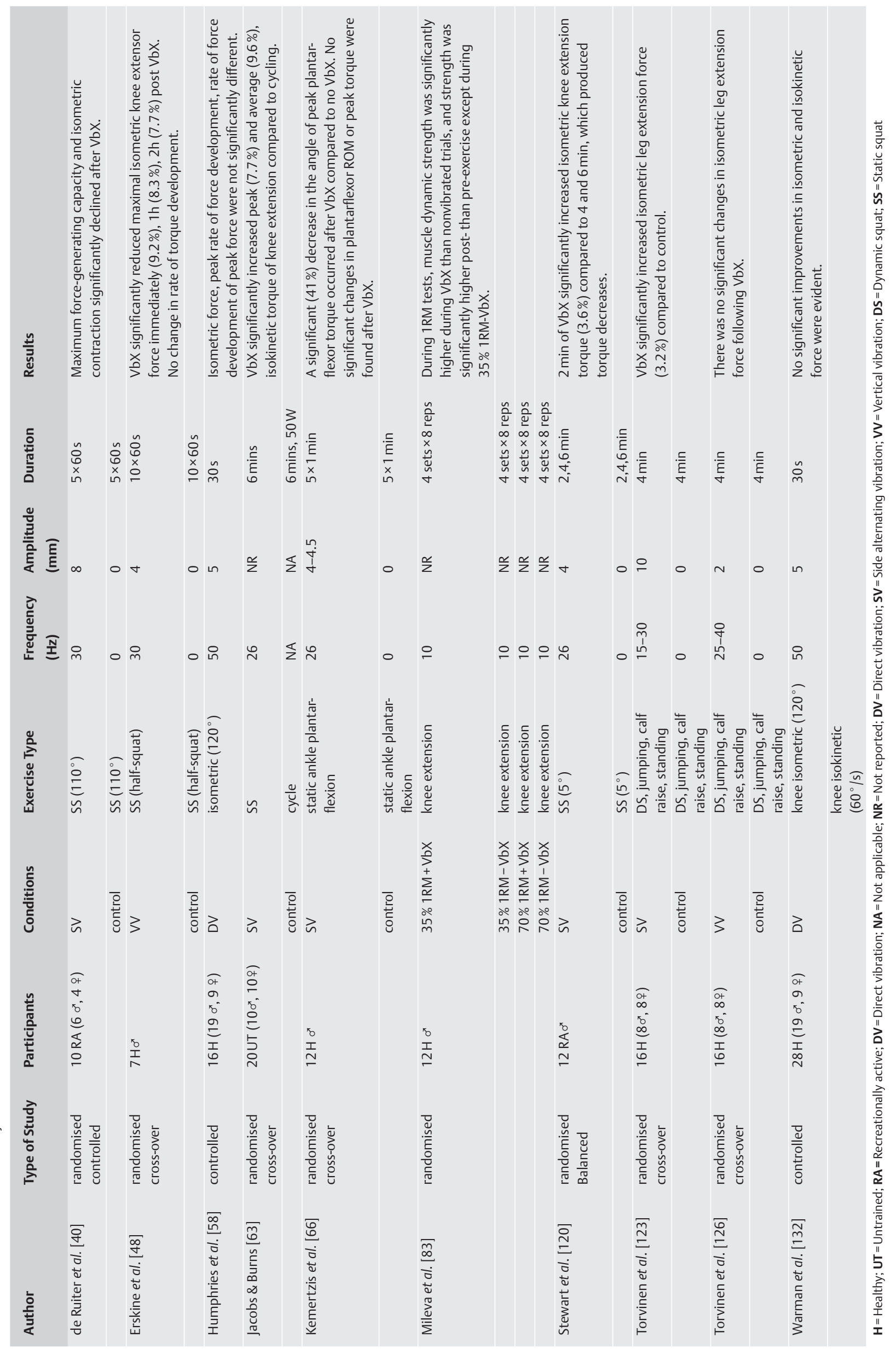


The discrepancy of the above findings could be due to the various protocols used for testing vibration. These include different methods of vibration, types and parameters of muscle contraction, and duration and frequency of vibration, as well as the muscle contraction velocities and variables.

\section{Short-term vibration ( $<2$ months) - Upper body force}

A summary of the studies that examined the short- and longterm effects of vibration on upper and lower body force can be found in 0 Table 8, 9. Using an electric motor to transmit eccentric oscillations ( $f=44 \mathrm{~Hz}, A=3 \mathrm{~mm}$ ) to a cable system a series of seated bench-pull repetitions were performed by male physical education students at $80-100 \% 1$ RM for 3 weeks (3x/week) while control groups performed the same resistance routine without vibration or performed calisthenics [59]. The researchers found that by combining vibration with force the 1RM benchpull significantly increased by $50 \%$ compared to a $16 \%$ improvement by the conventional resistance group, with no change being reported in the calisthenics group.

In a recent upper-body study by Silva et al. [119], untrained participants (24yrs) were either assigned to isometric bicep training (12 MVCs, $6 \mathrm{~s}$ in duration) without or with vibration $(f=8 \mathrm{~Hz}$, $A=6 \mathrm{~mm})$. The participants were seated with elbow flexed at $90^{\circ}$, and vibrations were produced by an amplifier connected to a steel cable with a hand grip that was applied in the opposite direction of muscle shortening. After 4 weeks of training (3 days/ week) there was a significant increase of $26 \%$ bicep MVC from the group that received isometric and vibration compared to isometric alone (10\% increase bicep MVC).

\section{Short-term vibration ( $<2$ months) - Lower body force}

Short-term vibration on lower-body force has produced mixed results with de Ruiter et al. [40,41] and Delecluse et al. [42] reporting no increase in muscle force from short-term vibration training. De Ruiter et al. [40] reported that MVC and maximal force-generating capacity with and without muscle stimulation of knee extensors were not enhanced after 11 weeks ( $3 x /$ week) of intermittent vibration $(f=30 \mathrm{~Hz}, A=8 \mathrm{~mm}$, SV [Galileo]). Delecluse et al. [42] found that 5 weeks of vibration $(f=35-40 \mathrm{~Hz}$, $A=1.7-2.5 \mathrm{~mm}$, VV [Power Plate]) failed to potentiate isometric and dynamic knee extensor and flexor strength in well-trained sprinters.

Increases in force from VbX have been reported from Mahieu et al. [77] and Fagnani et al. [49]; both studies observed an increase in torque post-vibration. Mahieu et al. [77] noted an increase in isokinetic torque of ankle plantar flexors of young skiers after 6 weeks ( $3 x /$ week) of vibration training $(f=24-28 \mathrm{~Hz}$, $A=2-4 \mathrm{~mm}, \mathrm{VV}$ [FitVibe]). Fagnani et al. [49] reported a $11.2 \%$ in isokinetic knee extensor in trained female athletes after 8 weeks (3x/week) intermittent vibration protocol $(f=35 \mathrm{~Hz}, A=4 \mathrm{~mm}$, VV [Nemes]). Both studies failed to compare vibration with the appropriate controls of performing the same activity with and without vibration. However, Ronnestad et al. [108] compared 5 weeks (2-3x/week) of weighted squats (6-10RM) with vibration $(f=40 \mathrm{~Hz}, A=$ not given $)$ and without vibration. They reported a $32 \%$ increase in 1 RM squat from vibration, but it was not significantly different from the $24 \%$ increase in 1 RM squat without vibration. The differences in vibration duration, amplitude, frequency, muscle groups, and vibration machines used in the aforementioned studies, may account for the discrepancy in results.

\section{Long-term vibration (>2 months) - Lower body force}

Torvinen et al. $[124,125]$ conducted 2 separate studies on the long-term effects of vibration $(f=25-35 \mathrm{~Hz}, A=2 \mathrm{~mm}$, VV [Kuntotary]) performed over 4 and 8 months in healthy young participants (19-38yrs). In the 4 month study [124] the authors found that isometric knee extensor strength improved by $3.7 \%$ at 2 months compared to the control condition, but no further improvements were evident at 4 months. Likewise, after 8 months [125] vibration had produced no significant differences in isometric knee extensor strength. Therefore, the vibration stimulus of frequency, amplitude, and duration may have not been effective in eliciting the desired neuromuscular responses. Ideally, the additional load should have been included in the latter stages of an 8 months' programme, which could have been achieved by progressively increasing body mass with external loads of a weighted vest or belt. Additionally, all of Torvenien's studies [123-126] have included an exercise routine of light squatting, light jumping and standing performed in addition to the vibration stimulus; however, this routine has never been quantified in terms of load or cadence. Similarly, the dynamic nature of the protocol could have inhibited the vibratory stimulus to realise its full potential.

To overcome the shortcomings of Torvinen's studies [123-126] Delecluse et al. [43] devised a 12 week (3x/week) periodised training plan, where vibration frequency, amplitude, exercise duration, load, sets and reps were progressively and systemically overloaded. 74 untrained females were randomly allocated to: 1) vibration ( $f=35-40 \mathrm{~Hz}, A=2.5-5 \mathrm{~mm}, a=2.3 \mathrm{~g}-5.1 \mathrm{~g}$ VV [Power Plate]); 2) cardio-resistance training; 3 ) placebo (very small amount of vibration $a=0.4 \mathrm{~g} \mathrm{VV}$, [Power Plate]); and 4) control. A $16 \%$ and $9 \%$ increase of isometric and dynamic knee force were observed in the vibration group, which was similar to the resistance training group (14\% and $7 \%$ respectively), but significantly different to placebo and control groups.

Roelants et al. [106] reported similar findings, that in older postmenopausal women (64yrs) isometric knee extensor strength increased by $15 \%$ and isokinetic strength by $16 \%$ from 24 weeks of vibration training $(f=35-40 \mathrm{~Hz}, A=2.5-5 \mathrm{~mm}$, VV [Power Plate]), however there was no significant difference in the respective increase found in the resistance training group. In a follow-up study using untrained females, this research group [69] confirmed their earlier findings of isometric and isokinetic knee extensor force, but they also noted that the increases were not significantly different from those who did a combined cardio and leg-strength programme.

The same research group [9] conducted a year-long study on older men (67 yrs) and repeated the same protocol, and observed that vibration increased muscle mass and isometric force but it was not significantly different from performing a combined cardio, strength, balance, and flexibility programme. Finally, Kvoring et al. [69] reported that after 9 weeks (2-3x/week) of weighted squats with vibration $(f=20-25 \mathrm{~Hz}, A=4 \mathrm{~mm}$, SV [Galileo 2000]) isometric leg press strength produced an increase of $9.3 \%$ which was comparable to the $12 \%$ increase in leg strength from weighted squats without vibration. Therefore, combining vibration with squats was no more beneficial than weighted squatting alone.

In summary, it is not conclusive whether acute vibration increases force attributes. This has been fraught by the type and parameters used for various muscle contractions, and the different sample populations that have varied in chronological age, experience and training status. Furthermore, the debate sur- 


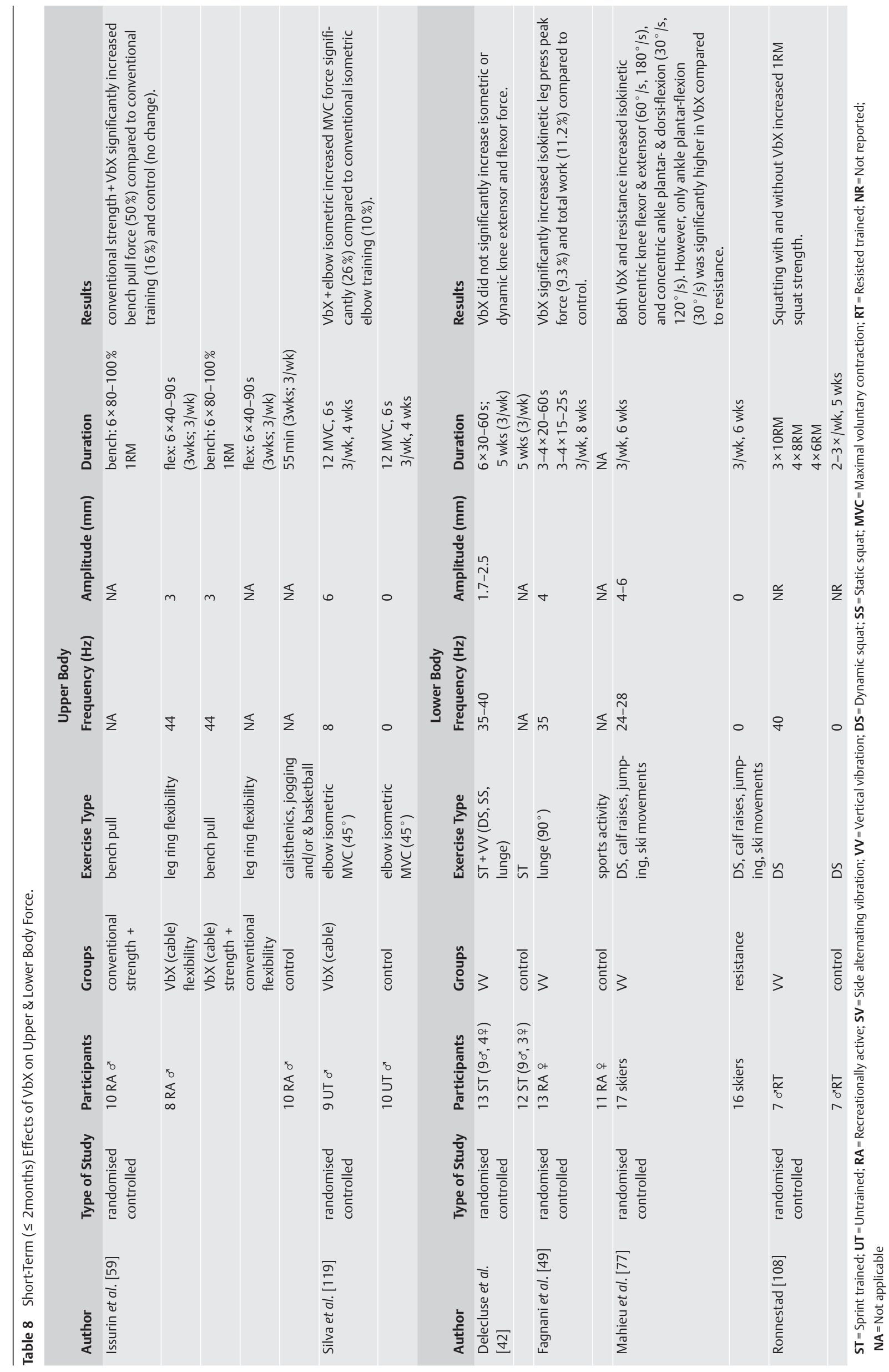



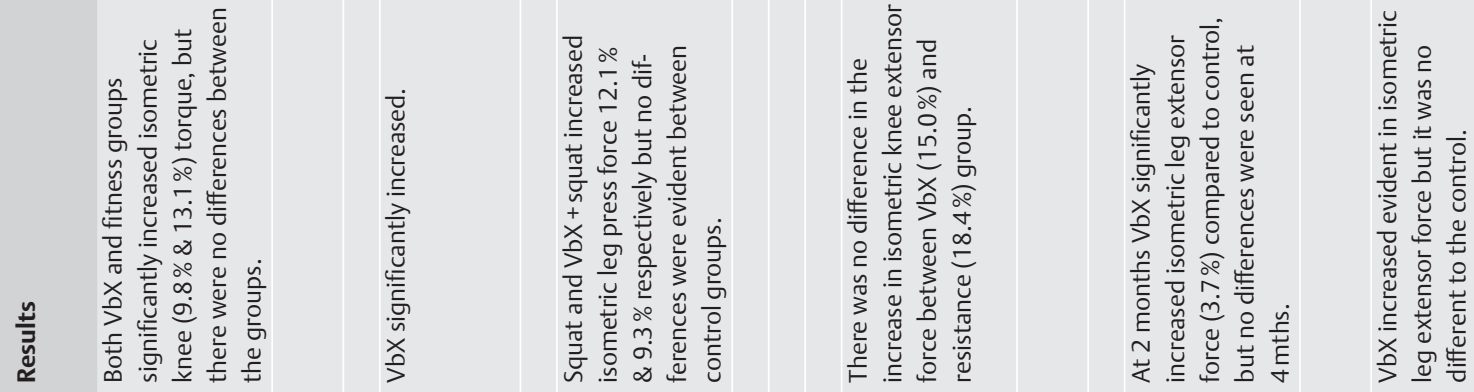

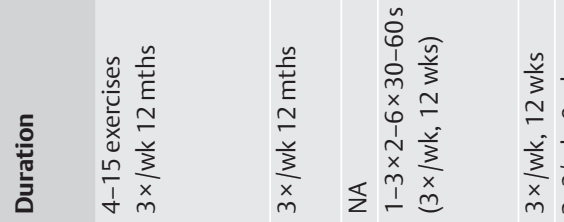

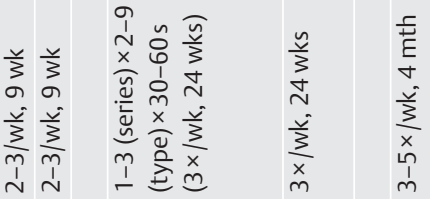

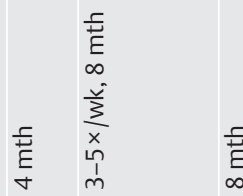

冚

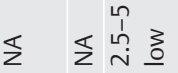

$+0 ะ \stackrel{\stackrel{n}{n}}{\sim}$
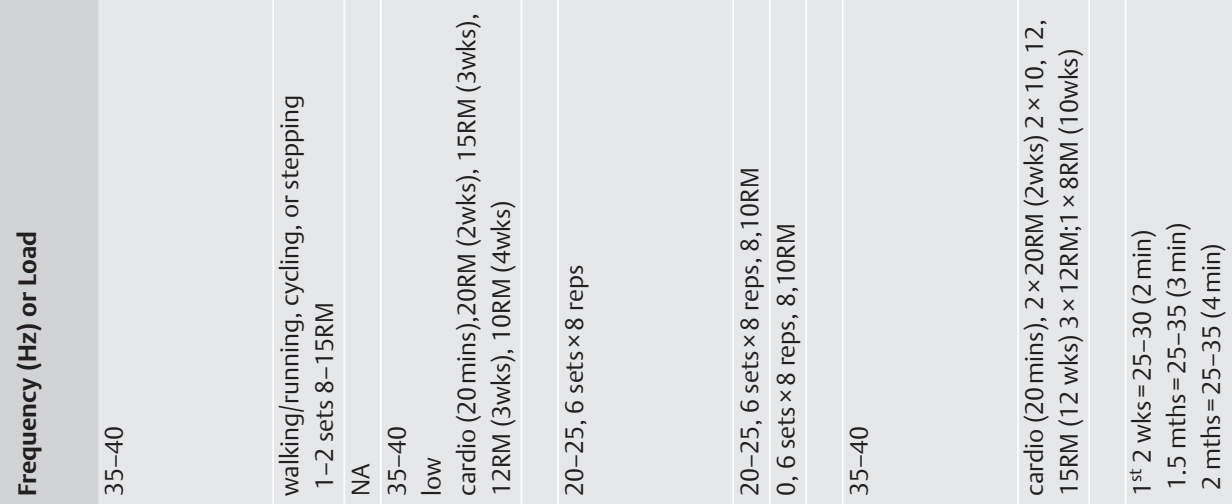

$\Sigma$
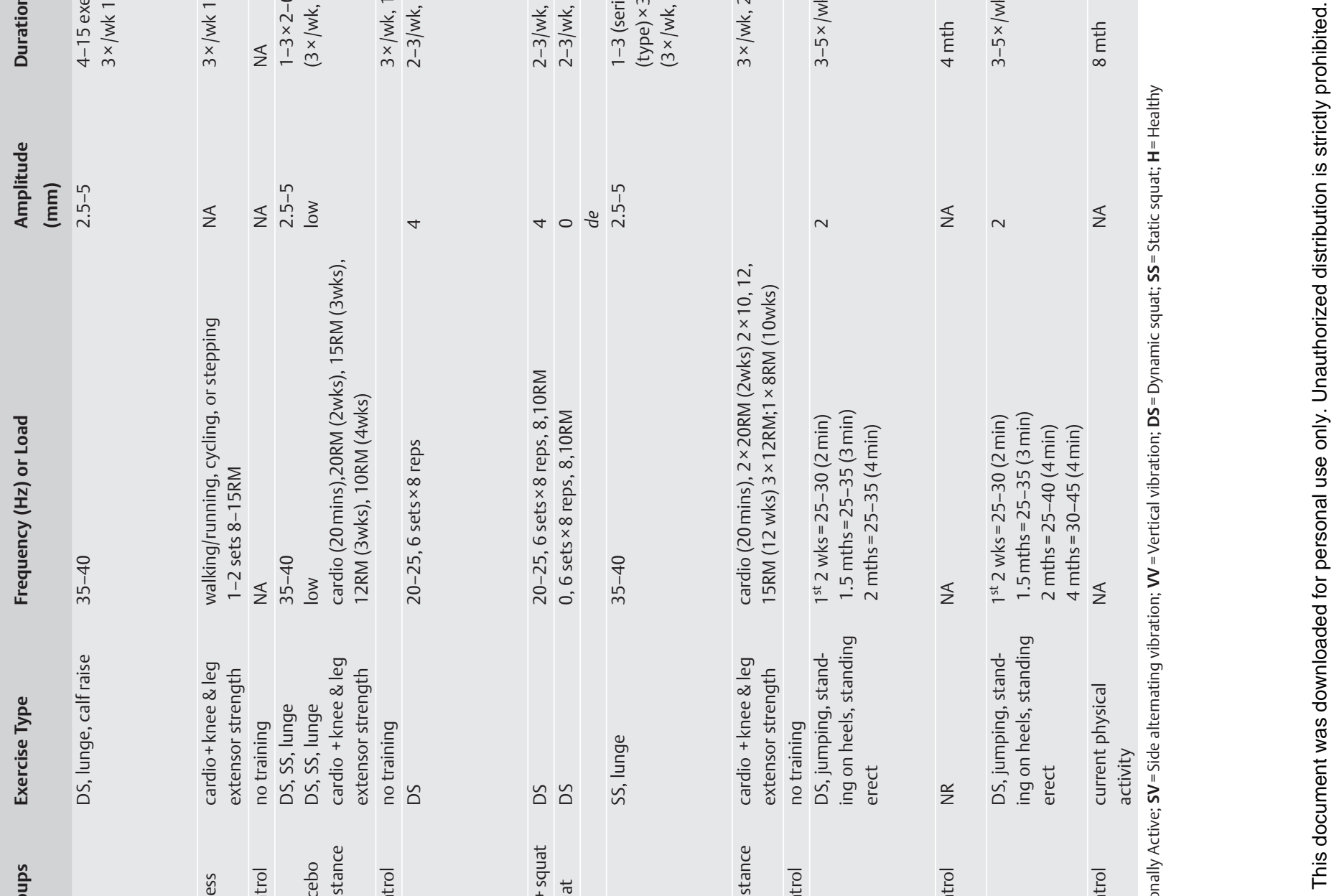

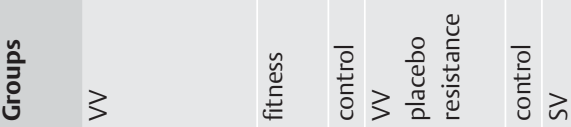

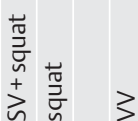

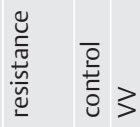

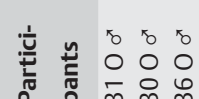

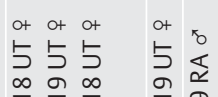

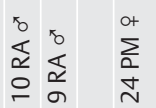

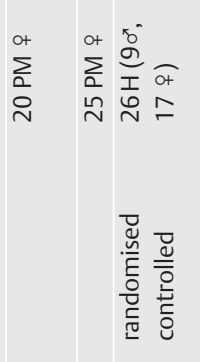

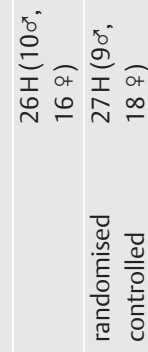

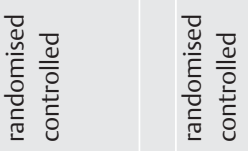

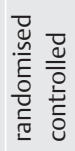

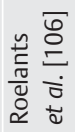

炡

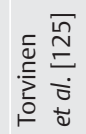

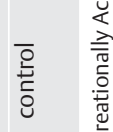


rounding the length-tension proposal that muscle must be lengthened in order to benefit from vibration has caused confusion. However, recent evidence suggests that when vibration was applied at $120^{\circ}$ knee flexion, which maximises the limb's greatest mechanical advantage, no increases in force were evident. Moreover, vibration applied to concentrically active muscle has shown to improve muscle force, which cannot explain the length-tension relationship. There may also be an optimal contraction velocity where vibration is most effective, and testing the effect of vibration on self-selected isokinetic contraction velocities warrants further investigation. Overall, acute $\mathrm{VbX}$ has a greater beneficial effect on power and force than short- and long-term studies, this could be due to the lack of knowledge surrounding the optimal method of periodising, loading, and progressing $\mathrm{VbX}$ variables in way that will sufficiently stimulate the musculoskeletal system. Currently there is a lack of scientific-based, short- and long-term VbX-training programmes. Secondly the acute exposure of $\mathrm{VbX}$ may provide a myogenic and neurogenic potentiation that reaches a threshold, which diminishes rapidly over an extended period of time, suggesting that $\mathrm{VbX}$ acts similar to a warm-up effect that may be promoted by post-activation potentiation.

\section{The effects of vibration exercise on compromised health \\ Multiple Sclerosis}

Multiple Sclerosis (MS) is a demyelinating disease of the central nervous system where decreases in power and strength can impair functional performance along with sensory losses, and visual disturbances. Shuhfried et al. [115] reported that MS patients undertaking acute intermittent $\mathrm{VbX}(f=2-4.4 \mathrm{~Hz}$, $A=3 \mathrm{~mm}$, [Zeptor-Med]) improved timed get up and go test compared to the placebo group that performed transcutaneous electrical nerve stimulation of the forearm, however no improvement in functional reach test was found. In a randomised cross over study where MS patients received 30 s of low frequency $(f=2 \mathrm{~Hz}$, $A=6 \mathrm{~mm}$ ) and high frequency ( $f=26, A=6 \mathrm{~mm}$, SV [FitVib]) there was a trend for higher torque in the quadriceps and hamstrings after $26 \mathrm{~Hz}$, compared to $2 \mathrm{~Hz}$, but they were not significantly different [61]. Using a counter-balance research design [116], MS patients were randomly allocated into 2 groups $(n=8)$ that received 3x/week for 4 weeks of strengthening and stretching exercises with or without vibration. Results found that $\mathrm{VbX}$ and exercise alone had a positive effect on isometric muscle force and well-being but there were no significance differences between the interventions. Likewise, the addition of vibration failed to enhance the functional measures of $10 \mathrm{~m}$ walk time and mobility (timed up and go) compared to conventional exercises. There are positive signs that VbX may have an effect on MS, however a long-term training study with appropriate controls is required to validate whether $\mathrm{VbX}$ is a viable exercise training option.

\section{Stroke}

Stroke can impact on motor function and impair balance, gait and reduce voluntary strength [122]. To date, 2 studies have reported favourable findings of using $\mathrm{VbX}$ for stroke patients. Using a force platform to measure the centre of pressure, postural control, Stroke patients improved their function from an intermittent exposure $(4 \times 45 \mathrm{~s})$ of $\mathrm{VbX}(f=30 \mathrm{~Hz}, A=3 \mathrm{~mm}$, SSV [Galileo 900]) [129]. Tihanyi et al. [122] found that an intermittent exposure $(6 \times 60 \mathrm{~s})$ of $\mathrm{VbX}(f=20 \mathrm{~Hz}, A=5 \mathrm{~mm}$, VV [Nemes]) improved isometric and eccentric knee extension torque, along with corresponding increase in EMG amplitude of the vastus lateralis compared to a control (no vibration). Conversely, a 6 week vibration study performed $5 \mathrm{x} /$ week of $4 \times 45 \mathrm{~s}(f=30 \mathrm{~Hz}, A=3 \mathrm{~mm}$, SSV [Galileo 900]) reported no significant differences in balance and functional activities between vibration group and those who performed exercise therapy to music [129].

\section{Parkinson's Disease}

Favourable results have been reported from patients suffering from Parkinson's disease (PD). Turbanski et al. [128] found that intermittent vibration $(5 \times 60 \mathrm{~s})$ exercise $(f=6 \mathrm{~Hz}, A=3 \mathrm{~mm}$, [Zeptor-Med]) improved postural stability of tandem standing as measured by Coordex platform system compared to control. Haas et al. [53], reported that PD patients exposed to intermittent $(5 \times 60 \mathrm{~s}) \mathrm{VbX}(f=6 \mathrm{~Hz}, A=1 \mathrm{~mm}$, Zeptor-Med system $)$ significantly improved their motor score by $17 \%$ from a rating scale (Unified Parkinson's Disease Rating Scale). However, the same investigator [52] found no improvement in proprioception from intermittent $\mathrm{VbX}(f=6 \mathrm{~Hz}, A=$ not given, SRT-medical system) compared to control. Conversely, Ebersbach et al. [47] reported a significant improvement in balance score (Tinetti Balance) from PD patients performing $\mathrm{VbX}(f=$ not given, irregular low frequency administered $A=$ not given, SV [Galileo 2000]) 2x/week for 3 weeks, however conventional balance training improved by the same margin.

\section{Age-related aspects of the elderly}

There have been positive effects of using $\mathrm{VbX}$ to improve balance in older people $[7,10,28,130]$. Recently Rees et al. [96] have reported benefits for older people ( $73 \mathrm{yrs}$ ) completing 2 months (3x/week) of $\mathrm{VbX}(f=26 \mathrm{~Hz}, A=5-8 \mathrm{~mm}$, SSV [Galileo]) with improvements in chair-rising time, timed-up-and-go test and faster walking speed, but, these increases were comparable to the exercise group that performed the same exercises without vibration. However, VbX did significantly increase ankle plantar flexion compared to the conventional exercise group but this did not correspond to enhancing physical performance. Kawanabe et al. [65] found that continuous (4 min) $\mathrm{VbX}(f=12-20 \mathrm{~Hz}$, $A=$ not given, SSV [Galileo]) performed $1 \mathrm{x} /$ week for 2 months significantly improved $10 \mathrm{~m}$ walk time, step length and the maximum standing time on one leg (right and left) in older participants (72 yrs) compared to an exercise routine performed without vibration. However, this experiment was not a randomised controlled study as the choice to have $\mathrm{VbX}$ was determined by the participants. Moreover the study lacked documentation on how the vibration frequency was progressed and the number of reps and sets that were performed by the participants. Bogaerts et al. [9] reported that in older males (60$80 \mathrm{yrs}$ ) isometric knee extensor strength, VJ height and muscle mass increased significantly by $9.8 \%, 10.9 \%$, and $3.4 \%$ after 12 months ( $3 \mathrm{x} /$ week) of VbX $(f=35-40 \mathrm{~Hz}, A=2.5-5 \mathrm{~mm}, \mathrm{VV}$, [Power Plate]). However, these increases were not significantly different from performing a combined cardiovascular, strength, balance, and flexibility programme conducted over the same duration.

\section{Postmenopausal women}

Long-term VbX studies ( $\geq 6$ months) have shown that balance, postural sway and muscle power is significantly enhanced in postmenopausal women $[51,110,130]$. Additionally, isometric (knee joint $\left.=130^{\circ}\right)$, isokinetic strength $\left(100^{\circ} / \mathrm{s}\right)$ and VJ height have been reported to increase after 6 months of $\mathrm{VbX}$ 
( $f=35-40 \mathrm{~Hz}, \mathrm{VV}$, [Power Plate]), in postmenopausal women $[106,130]$. Raimundo et al. [95] conducted an 8 month study where 27 postmenopausal women (66 yrs) were randomly assigned to $\operatorname{VbX}(f=12.6 \mathrm{~Hz}, A=6 \mathrm{~mm}$, Galileo [SSV] $)$ or a walk programme (60 min, $70-75 \% \mathrm{MHR}$ ) performed $3 \mathrm{x} /$ week. The researchers reported that the walk programme significantly improved $4 \mathrm{~m}$ walk time, and chair rise test more than $\mathrm{VbX}$, however VbX had a significant effect of increasing VJ, but neither intervention improved knee isokinetic strength. This finding is not surprising given that only a low frequency dose was used during the 8 months and the vibration frequency was not progressively increased over the course of the study.

\section{Other}

VbX $(f=20-25 \mathrm{~Hz}, A=3 \mathrm{~mm}$, SSV [Galileo 2000]) performed $5 \mathrm{x} /$ week for 3 months has been shown to improve muscular performance in Cystic Fibrosis (CF) of chair rising time, peak jump force and velocity, but no changes were found in forced expiratory volume and forced vital capacity [99]. In women with Fibromyalgia a significant improvement in pain and fatigue was recorded from receiving intermittent $\operatorname{VbX}(f=30 \mathrm{~Hz}, A=2 \mathrm{~mm}$, VV [Power Plate]) 2x/week for 6 weeks compared to exercise (salsa dancing, stretching and relaxation techniques) and control groups.

In a recent study conducted by Lauper et al. [71] they investigated pelvic floor stimulation using 2 different types of vibration platforms, SV (Galileo 900) and stochastic resonance vibration (SRV) (Zeptor-Med) which has 2 separate footplates which vibrate vertically and independently. Using an intravaginal surface EMG electrode the researchers reported that the SRV $(f=2$, $4,6,8,10,12 \mathrm{~Hz}, A=3 \mathrm{~mm}$ ) activated the pelvic floor muscles significantly more than SV $(f=5,15,25 \mathrm{~Hz}, A=2 \& 4 \mathrm{~mm})$ and initiated a higher pelvic floor activation than maximum contraction alone in post-partum compared to healthy controls. However, it is unknown whether this increase in muscle activation can lead to an increase in functional performance and whether symptoms of poor pelvic floor activation can be alleviated.

It is unclear if $\mathrm{VbX}$ is beneficial for those with osteoarthritis as there has been limited research conducted in this area. Trans et al. [127] randomly allocated patients suffering from knee osteoarthritis to $\mathrm{VbX}$, conventional balance and control groups. The results showed a non-significant $(\mathrm{p}=0.051)$ improvement in proprioception from intermittent $(6-9 \times 30-70 s)$ VbX $(f=25-$ $30 \mathrm{~Hz}, A=$ not given, [VibM]) with an increase in knee extensor strength. But those performing balance board with an implanted vibratory device showed a significant increase in proprioception, with no knee strength increases.

There is some evidence to suggest that cardiovascular or aerobic exercise may be able to enhance glycemic control in type II diabetics $[89,117]$, and that resistance training may be a possible treatment to fight metabolic diseases $[17,27,46]$. Di Loreto et al. [45] found that intermittent $\mathrm{VbX}(f=30 \mathrm{~Hz}, A= \pm 4 \mathrm{~mm}, \mathrm{VV}$, [Nemes]) decreased plasma glucose levels which may indicate that glucose is transported into the muscle by $\mathrm{VbX}$, which may be of benefit to diabetics. In a study that randomised type 2 diabetics into resistance, vibration, and flexibility groups, the researchers reported that intermittent $\mathrm{VbX}(f=30-35 \mathrm{~Hz}$, $A=2 \mathrm{~mm}$, VV [VibroGym]) performed 3x/week for 12 weeks had no effect on isometric knee extension, while fasting glucose levels, plasma glucose concentration from an oral ingestion of $75 \mathrm{~g}$ of glucose drink and haemoglobin showed reductions but were not significant from baseline measures [6].
In conclusion, $\mathrm{VbX}$ is a safe modality to increase reflex and muscle activity, and muscle performance in athletes, the aged and compromised health. However, there seems little benefit of using $\mathrm{VbX}$ to enhance cardiovascular indices, where VbX cannot increase heart rate to the same extent as conventional aerobic exercise. Despite its wide use there remain gaps of knowledge on aspects such as mechanism of action, clinical effects, and even details of regimens for particular therapeutic use. There is some dissonance that exists between various studies which can be explained by the different types of vibration platforms (SV, $\mathrm{VV}$ ), the participant characteristics (body/muscle mass, training status, muscle strength and stiffness, age, gender), the different permutations of vibration parameters (amplitude, exposure duration, rest interval) and the prescription (number of repetitions, sets, exercises). In summary, vibration is a harmless exercise modality that has the potential to benefit sport, exercise and health; likewise it can be used to compliment other modalities to enhance muscle activity and function and compromised health.

\section{References}

1 Abercromby AFJ, Amonette WE, Layne CS, McFarlin BK, Hinman MR, Paloski $W H$. Variation in neuromuscular responses during acute whole-body vibration exercise. Med Sci Sports Exerc 2007; 39: 1642-1650

2 Abercromby AFJ, Amonette WE, Layne CS, McFarlin BK, Hinman MR, Paloski $W H$. Vibration exposure and biodynamic responses during whole-body vibration training. Med Sci Sports Exerc 2007; 39: 1794-1800

3 Adams JB, Edwards D, Serviette D, Bedient A, Huntsman E, Jacobs KA, Del Rossi G, Roos BA, Signorile JF. Optimal frequency, displacment, duration, and recovery patterns to maximise power output following acute whole-body vibration. J Strength Cond Res 2009; 23: $237-245$

4 Annino G, Padua E, Castagna C, Di Salvo V, Minichella S, Tsarpela O, Manzi V, D'Ottavio $S$. Effect of whole body vibration training on lower limb performance in selected high-level ballet student. J Strength Cond Res 2007; 21: 1072-1076

5 Armstrong WJ, Nestle HN, Grinnell DC, Cole LD, Van Gilder EL, Warren GS, Capizzi EA. The acute effect of whole-body vibration on the Hoffmann reflex. J Strength Cond Res 2008; 22: 471-476

6 Baum K, Votteler T, Schiab J. Efficiency of vibration exercise for glycemic control in type 2 diabetes patients. Int J Med Sci 2007; 4: 159-163

7 Bautmans I, Van Hees E, Lemper J-C, Mets T. The feasibility of whole body vibration in institutionalised elderly persons and its influence on muscle performance, balance, and mobility: a randomised controlled trial. BMC Geriatr 2005; 5: 1-8

8 Bazett-Jones DM, Finch HW, Dugan EL. Comparing the effects of various whole-body vibration accelerations on counter-movement jump performance. J Sports Sci Med 2008; 7: 144-150

9 Bogaerts A, Delecluse C, Claessens AL, Coudyzer W, Boonen S, Verschueren SMP. Impact of whole-body vibration training versus fitness training on muscle strength and muscle mass in older men: A 1-year randomized controlled trial. J Gerontol A Biol Sci Med Sci 2007; 62: 630-635

10 Bogaerts A, Verschueren S, Delecluse C, Claessens AL, Boonen S. Effects of whole body vibration training on postural control in older individuals: A 1 year randomized controlled trial. Gait Posture 2007; 26: 309-316

11 Bongiovanni $L G$, Hagbarth $K E$. Tonic vibration reflexes elicited during fatigue from maximal voluntary contractions in man. J Physiol 1990; 423: $1-14$

12 Bosco C, Cardinale M, Tsarpela O. Influence of vibration on mechanical power and electromyogram activity in human arm flexor muscles. Eur J Appl Physiol 1999; 79: 306-311

13 Bosco C, Cardinale M, Tsarpela O, Colli R, Tihanyi J, Duvillard SP, Viru $A$. The influence of whole body vibration on jumping performance. Biol Sport 1998; 15: 157-164

14 Bosco C, Colli R, Introini E, Cardinale M, Tsarpela O, Madella A, Tihanyi $J$, Viru A. Adaptive responses of human skeletal muscle to vibration exposure. Clin Physiol 1999; 19: 183-187 
15 Bosco C, Iacovelli M, Tsarpela O, Viru A. Hormonal responses to whole body vibration in men. Eur J Appl Physiol 2000; 81: 449-454

16 Broadbent S, Rousseau J, Thorp RM, Choate SL, Jackson FS, Rowlands $D S$. Vibration therapy reduces plasma IL-6 and muscle soreness after downhill running. Br J Sports Med 2008, doi:10.1136/bjsm052100

17 Brooks N, Layne JE, Gordon PL, Roubenoff R, Nelson ME, CastanedaSceppa C. Strength training improves muscle quality and insulin sensitivity in Hispanic older adults with type 2 diabetes. Int J Med Sci 2007; 4: 19-27

18 Bullock N, Martin DT, Ross A, Rosemond CD, Jordan MJ, Marino FE. Acute effect of whole-body vibration on sprint and jumping performance in elite skeleton athletes. J Strength Cond Res 2008; 22: 1371-1374

19 Burke JR, Schutten MC, Koceja DM, Kamen G. Age-dependent effects of muscle vibration and the Jendrassik maneuver on the patellar tendon reflex response. Arch Phys Med Rehabil 1996; 77: 600-604

20 Button C, Anderson N, Bradford C, Cotter JD, Ainslie PN. The effect of multidirectional mechanical vibration on peripheral circulation of humans. Clin Physiol Funct Imaging 2007; 27: 211-216

21 Cardinale $M$, Bosco $C$. The use of vibration as an exercise intervention. Exerc Sport Sci Rev 2003; 31: 3-7

22 Cardinale M, Erskine JA. Vibration training in elite sport: effective training solution or just another fad? Int J Sports Physiol Perform 2008; 3: 232-239

23 Cardinale M, Ferrari M, Quaresima V. Gastrocnemius medialis and vastus lateralis oxygenation during whole-body vibration exercise. Med Sci Sports Exerc 2007; 39: 694-700

24 Cardinale M, Leiper J, Erskine J, Milroy M, Bell S. The acute effects of different whole body vibration amplitudes on the endocrine system of young healthy men: a preliminary study. Clin Physiol Funct Imaging 2006; 26: 380-384

25 Cardinale M, Lim J. Electromyography activity of vastus lateralis muscle during whole-body vibrations of different frequencies. J Strength Cond Res 2003; 17: 621-624

26 Cardinale M, Wakeling J. Whole body vibration exercise: are vibrations good for you? Br J Sports Med 2005; 39: 585-589

27 Cauza E, Hanusch-Enserer U, Strasser B, Ludvik B, Metz-Schimmerl S, Pacini G, Wagner O, Georg P, Prager R, Kostner K, Dunky A, Haber P. The relative benefits of endurance and strength training on the metabolic factors and muscle function of people with type 2 diabetes mellitus. Arch Phys Med Rehabil 2005; 86: 1527-1533

28 Cheung WH, Mok HW, Qin L, Sze PC, Lee KM, Leung KS. High-frequency whole-body vibration improves balancing ability in elderly women. Arch Phys Med Rehabil 2007; 88: 852-857

29 Cochrane DJ, Hawke EJ. Effects of acute upper-body vibration on strength and power variables in climbers. J Strength Cond Res 2007; 21: $527-531$

30 Cochrane DJ, Legg SJ, Hooker MJ. The short-term effect of whole-body vibration training on vertical jump, sprint, and agility performance. J Strength Cond Res 2004; 18: 828-832

31 Cochrane DJ, Sartor F, Winwood K, Stannard SR, Narici M, Rittweger $J$. A comparison of the acute physiologic effects of acute whole-body vibration exercise in young and older people. Arch Phys Med Rehabil 2008; 89: 815-821

32 Cochrane DJ, Stannard SR. Acute whole body vibration training increases vertical jump and flexibility performance in elite female field hockey players. Br J Sports Med 2005; 39: 860-865

33 Cochrane DJ, Stannard SR, Firth EC, Rittweger J. Acute whole-body vibration elicits post-activation potentiation. Eur J Appl Physiol 2010; 108: 311-319

34 Cochrane DJ, Stannard SR, Sargeant T, Rittweger J. The rate of muscle temperature increase during acute whole-body vibration exercise. Eur J Appl Physiol 2008; 103: 441-448

35 Colson SS, Petit P-D, Hebreard L, Tessaro J, Pensini M. Whole-body vibration does not enhance muscle activaton. Int J Sports Med 2009, In Press

36 Cormie P, Deane RS, Triplett NT, McBride JM. Acute effects of wholebody vibration on muscle activity, strength, and power. J Strength Cond Res 2006; 20: 257-261

37 Crewther B, Cronin J, Keogh J. Gravitational forces and whole body vibration: implications for prescription of vibratory stimulation. Phys Ther Sport 2004; 5: 37-43

38 Cronin JB, Oliver M, McNair PJ. Muscle stiffness and injury effects of whole body vibration. Phys Ther Sport 2004; 5: 68-74

39 Da Silva ME, Nunez VM, Vaamonde D, Fernandez JM, Poblador MS, Garcia-Manso JM, Lancho JL. Effects of different frequencies of whole body vibration on muscular performance. Biol Sport 2006; 23: $267-282$
40 de Ruiter CJ, van der Linden RM, van der Zijden MJA, Hollander AP, de Haan A. Short-term effects of whole-body vibration on maximal voluntary isometric knee extensor force and rate of force rise. Eur J Appl Physiol 2003; 88: 472-475

41 de Ruiter CJ, van Raak SM, Schilperoort JV, Hollander AP, de Haan A. The effects of 11 weeks whole body vibration training on jump height, contractile properties and activation of human knee extensors. Eur J Appl Physiol 2003; 90: 595-600

42 Delecluse C, Roelants M, Diels R, Koninckx E, Verschueren S. Effects of whole body vibration training on muscle strength and sprint performance in sprint-trained athletes. Int J Sports Med 2005; 26: 638-644

43 Delecluse C, Roelants $M$, Verschueren S. Strength increase after wholebody vibration compared with resistance training. Med Sci Sports Exerc 2003; 35: 1033-1041

44 Di Giminiani R, Tihanyi J, Safar S, Scrimaglio R. The effects of vibration on explosive and reactive strength when applying individualized vibration frequencies. J Sports Sci 2009; 27: 169-177

45 Di Loreto C, Ranchelli A, Lucidi P, Murdolo G, Parlanti N, De Cicco A, Tsarpela O, Annino G, Bosco C, Santeusanio F, Bolli GB, De Feo P. Effects of whole-body vibration exercise on the endocrine system of healthy men. J Endocrinol Invest 2004; 27: 323-327

46 Dunstan DW, Daly RM, Owen N, Jolley D, De Courten M, Shaw J, Zimmet $P$. High-intensity resistance training improves glycemic control in older patients with type 2 diabetes. Diabetes Care 2002; 25 : 1729-1736

47 Ebersbach G, Edler D, Kaufhold O, Wissel J. Whole body vibration versus conventional physiotherapy to improve balance and gait in Parkinson's disease. Arch Phys Med Rehabil 2008; 89: 399-403

48 Erskine J, Smillie I, Leiper J, Ball D, Cardinale M. Neuromuscular and hormonal responses to a single session of whole body vibration exercise in healthy young men. Clin Physiol Funct Imaging 2007; 27: $242-248$

49 Fagnani F, Giombini A, Di Cesare A, Pigozzi F, Di Salvo V. The effects of a whole-body vibration program on muscle performance and flexibility in female athletes. Am J Phys Med Rehab 2006; 85: 956-962

50 Gregory JE, McIntyre AK, Proske U. Tendon organ afferents in the knee-joint nerve of the cat. Neurosci Lett 1989; 103: 287-292

51 Gusi $N$, Raimundo A, Leal A. Low-frequency vibratory exercise reduces the risk of bone fracture more than walking: a randomized controlled trial. BMC Musculoskelet Disord 2006; 30: 92

52 Haas CT, Buhlmann A, Turbanski S, Schmidtbleicher D. Proprioceptive and sensorimotor performance in Parkinson's disease. Res Sports Med 2006; 14: 273-287

53 Haas CT, Turbanski S, Kaiser I, Schmidtbleicher D. Influences of wholebody-vibration on symptom structure in Parkinson's disease. J Neurol 2004; 251: 18-18

54 Hazell TJ, Jakobi JM, Kenno KA. The effects of whole-body vibration on upper- and lower-body EMG during static and dynamic contractions. Appl Physiol Nutr Metab 2007; 32: 1156-1163

55 Hazell TJ, Thomas GWR, DeGuire JR, Lemon PWR. Vertical whole-body vibration does not increase cardiovascular stress to static semi-squat exercise. Eur J Appl Physiol 2008; 104: 903-908

56 Hodgson $M$, Docherty D, Robbins D. Post-activation potentiation Underlying physiology and implications for motor performance. Sports Med 2005; 35: 585-595

57 Hopkins JT, Fredericks D, Guyon PW, Parker S, Gage M, Feland JB, Hunter $I$. Whole body vibration does not potentiate the stretch reflex. Int J Sports Med 2008; 30: 124-129

58 Humphries B, Warman G, Purton J, Doyle T, Dugan E. The influence of vibration on muscle activation and rate of force development during maximal isometric contractions. J Sports Sci Med 2004; 3: $16-22$

59 Issurin VB, Liebermann DG, Tenenbaum G. Effect of vibratory stimulation training on maximal force and flexibility. J Sports Sci 1994; 12 : 561-566

60 Issurin VB, Tenenbaum G. Acute and residual effects of vibratory stimulation on explosive strength in elite and amateur athletes. J Sports Sci 1999; 17: 177-182

61 Jackson KJ, Merriman HL, Vanderburgh PM, Brahler CJ. Acute effects of whole-body vibration on lower extremity muscle performance in persons with multiple sclerosis. J Neurol Phys Ther 2008; 32: 171-176

62 Jackson SW, Turner DL. Prolonged muscle vibration reduces maximal voluntary knee extension performance in both the ipsilateral and the contralateral limb in man. Eur J Appl Physiol 2003; 88: 380-386 
63 Jacobs PL, Burns $P$. Acute enhancement of lower-extremity dynamic strength and flexibility with whole-body vibration. J Strength Cond Res 2008; 23: 51-57

64 Jordan $M$, Norris S, Smith D, Herzog W. Acute effects of whole-body vibration on peak isometric torque, muscle twitch torque and voluntary muscle activation of the knee extensors. Scand J Med Sci Sports 2010; 20: 535-540

65 Kawanabe K, Kawashima A, Sashimoto I, Takeda T, Sato Y, Iwamoto J. Effect of whole-body vibration exercise and muscle strengthening, balance, and walking exercises on walking ability in the elderly. Keio J Med 2007; 56: 28-33

66 Kemertzis MA, Lythgo ND, Morgan DL, Galea MP. Ankle flexors produce peak torque at longer muscle lengths after whole-body vibration. Med Sci Sports Exerc 2008; 40: 1977-1983

67 Kerschan-Schindl K, Grampp S, Henk C, Resch H, Preisinger E, FialkaMoser $V$, Imhof $H$. Whole-body vibration exercise leads to alterations in muscle blood volume. Clin Physiol 2001; 21: 377-382

68 Kin-Isler A, Acikada C, Aritan S. Effects of vibration on maximal isometric muscle contraction at different joint angles. Isokinet Exerc Sci 2006; 14: 213-220

69 Kvorning T, Bagger M, Caserotti P, Madsen K. Effects of vibration and resistance training on neuromuscular and hormonal measures. Eur J Appl Physiol 2006; 96: 615-625

70 Lafortune MA, Lake MJ, Hennig EM. Differential shock transmission response of the human body to impact severity and lower limb posture. J Biomech 1996; 29: 1531-1537

71 Lauper M, Kuhn A, Gerber R, Luginbuhl H, Radlinger L. Pelvic floor stimulation: What are the good vibrations? Neurourol Urodyn 2009; 28: $405-410$

72 Lohman EB, Scott PJ, Maloney-Hinds C, Betts-Schwab H, Thorpe D. The effect of whole body vibration on lower extremity skin blood flow in normal subjects. Med Sci Monit 2007; 13: 71-76

73 Lorenzen C, Maschette $W$, Koh $M$, Wilson $C$. Inconsistent use of terminology in whole body vibration exercise research. J Sci Med Sport 2009; 12: 676-678

74 Luo J, Clarke M, McNamara B, Moran K. Influence of resistance load on neuromuscular response to vibration training. J Strength Cond Res 2009; 23: 420-426

75 Luo J, McNamara B, Moran K. Effect of vibration training on neuromuscular output with ballistic knee extensions. J Sports Sci 2008; 26: 1365-1373

76 Lythgo N, Eser P, de Groot P, Galea $M$. Whole-body vibration dosage alters leg blood flow. Clin Physiol Funct Imaging 2009; 29: 53-59

77 Mahieu NN, Witvrouw E, Van de Voorde D, Michilsens D, Arbyn V, Van den Broecke $W$. Improving strength and postural control in young skiers: Whole-body vibration versus equivalent resistance training. J Athl Training 2006; 41: 286-293

78 Maloney-Hinds C, Petrofsky JS, Zimmerman G. The effect of $30 \mathrm{~Hz}$ vs. $50 \mathrm{~Hz}$ passive vibration and duration of vibration on skin blood flow in the arm. Med Sci Monit 2008; 14: CR112-CR116

79 McBride JM, Nuzzo JL, Dayne AM, Israetel MA, Nieman DC, Triplett NT. Effect of an acute bout of whole body vibration exercise on muscle force output and motor neuron excitability. J Strength Cond Res 2010; 24: 184-189

80 McCloskey DI, Matthews PB, Mitchell JH. Absence of appreciable cardiovascular and respiratory responses to muscle vibration. J Appl Physiol 1972; 33: 623-626

81 Melnyk M, Kofler B, Faist M, Hodapp M, Gollhofer A. Effect of a wholebody vibration session on knee stability. Int J Sports Med 2008; 29: 839-844

82 Mester J, Spitzenpfeil P, Schwarzer J, Seifriz F. Biological reaction to vibration - implications for sport. J Sci Med Sport 1999; 2: 211-226

83 Mileva KN, Naleem AA, Biswas SK, Marwood S, Bowtell JL. Acute effects of a vibration-like stimulus during knee extension exercise. Med Sci Sports Exerc 2006; 38: 1317-1328

84 Mischi $M$, Cardinale $M$. The effects of a $28-\mathrm{Hz}$ vibration on arm muscle activity during isometric exercise. Med Sci Sports Exerc 2009; 41: 645-652

85 Moran K, McNamara B, Luo J. Effect of vibration training in maximal effort (70\% 1RM) dynamic bicep curls. Med Sci Sports Exerc 2007; 39: $526-533$

86 Nazarov V, Spivak G. Development of athlete's strength abilities by means of biomechanical stimulation method. Theo Prac Phys Cult (Moscow) 1985; 12: 445-450

87 Nigg BM, Wakeling JM. Impact forces and muscle tuning: a new paradigm. Exerc Sport Sci Rev 2001; 29: 37-41
88 Nishihira $Y$, Iwaski T, Hatta A, Wasaka T, Kanada T, Kuroiwa K, Akiyawa S, Kida T, Ryol K. Effect of whole body vibration stimulus and voluntary contraction on motoneuron pool. Adv Exerc Sports Physiol 2002; 8: 83-86

89 Ostergard T, Andersen JL, Nyholm B, Lund S, Nair KS, Saltin B, Schmitz 0 . Impact of exercise training on insulin sensitivity, physical fitness, and muscle oxidative capacity in first-degree relatives of type 2 diabetic patients. Am J Physiol Endocrinol Metab 2006; 290: 998-1005

90 Otsuki T, Takanami Y, Aoi W, Kawai Y, Ichikawa H, Yoshikawa T. Arterial stiffness acutely decreases after whole-body vibration in humans. Acta Physiol 2008; 194: 189-194

91 Pel JJM, Bagheri J, van Dam LM, van den Berg-Emons HJG, Horemans $H L D$, Stam HJ, van der Steen J. Platform accelerations of three different whole-body vibration devices and the transmission of vertical vibrations to the lower limbs. Med Eng Phys 2009; 31: 937-944

92 Pellegrini MJ, Lythgo ND, Morgan DL, Galea MP. Voluntary activation of the ankle plantar flexors following whole-body vibration. Eur J Appl Physiol 2010; 108: 927-934

93 Pierrot-Deseilligny E, Mazevet D. The monosynaptic reflex: a tool to investigate motor control in humans. Interest and limits. Neurophysiol Clin 2000; 30: 67-80

94 Poston B, Holcomb WR, Guadagnoli MA, Linn LL. The acute effects of mechanical vibration on power output in the bench press. J Strength Cond Res 2007; 21: 199-203

95 Raimundo AM, Gusi N, Tomas-Carus P. Fitness efficacy of vibratory exercise compared to walking in postmenopausal women. Eur J Appl Physiol 2009; 106: 741-748

96 Rees S, Murphy A, Watsford M. Effects of vibration exercise on muscle performance and mobility in an older population. J Aging Phys Act 2007; 15: 367-381

97 Rehn B, Lidstrom J, Skoglund J, Lindstrom B. Effects on leg muscular performance from whole-body vibration exercise: a systematic review. Scand J Med Sci Sports 2007; 17: 2-11

98 Rhea MR, Kenn JG. The effect of acute applications of whole-body vibration on the itonic platform on subsequent lower-body power output during the back squat. J Strength Cond Res 2009; 23: 58-61

99 Rietschela E, van Koningsbruggena S, Frickeb O, Semlerb O, Schoenaub $E$. Whole body vibration: a new therapeutic approach to improve muscle function in cystic fibrosis? Int J Rehabil Res 2008; 31: 253-256

100 Rittweger J. Vibration as an exercise modality: how it may work and what its potential might be. Eur J Appl Physiol 2010; 108: 877-904

101 Rittweger J, Beller G, Felsenberg D. Acute physiological effects of exhaustive whole-body vibration exercise in man. Clin Physiol 2000; 20: 134-142

102 Rittweger J, Ehrig J, Just K, Mutschelknauss M, Kirsch KA, Felsenberg $D$. Oxygen uptake in whole-body vibration exercise: influence of vibration frequency, amplitude, and external load. Int J Sports Med 2002; 23: 428-432

103 Rittweger J, Mutschelknauss M, Felsenberg D. Acute changes in neuromuscular excitability after exhaustive whole body vibration exercise as compared to exhaustion by squatting exercise. Clin Physiol Funct Imaging 2003; 23: 81-86

104 Rittweger J, Schiessl H, Felsenberg D. Oxygen uptake during wholebody vibration exercise: comparison with squatting as a slow voluntary movement. Eur J Appl Physiol 2001; 86: 169-173

105 Roelants M, Delecluse C, Goris M, Verschueren S. Effects of 24 weeks of whole body vibration training on body composition and muscle strength in untrained females. Int J Sports Med 2004; 25: 1-5

106 Roelants $M$, Delecluse C, Verschueren SM. Whole-body-vibration training increases knee-extension strength and speed of movement in older women. J Am Geriatr Soc 2004; 52: 901-908

107 Roelants M, Verschueren SMP, Delecluse C, Levin O, Stijnen V. Wholebody-vibration-induced increase in leg muscle activity during different squat exercises. J Strength Cond Res 2006; 20: 124-129

108 Ronnestad BR. Comparing the performance-enhancing effects of squats on a vibration platform with conventional squats in recreationally resistance-trained men. J Strength Cond Res 2004; 18: 839-845

109 Rubin C, Pope M, Fritton JC, Magnusson M, Hansson T, McLeod K. Transmissibility of 15-hertz to 35-hertz vibrations to the human hip and lumbar spine: Determining the physiologic feasibility of delivering low-level anabolic mechanical stimuli to skeletal regions at greatest risk of fracture because of osteoporosis. Spine 2003; 28 . 2621-2627 
110 Russo CR, Lauretani F, Bandinelli S, Bartali B, Cavazzini C, Guralnik $J M$, Ferrucci $L$. High-frequency vibration training increases muscle power in postmenopausal women. Arch Phys Med Rehabil 2003; 84: $1854-1857$

111 Sale DG. Postactivation potentiation: role in human performance. Exerc Sport Sci Rev 2002; 30: 138-143

112 Sands WA, McNeal JR, Stone MH, Haff GG. Effect of vibration on forward split flexibility and pain perception in young male gymnasts. Int J Sports Physiol Perform 2008; 3: 469-481

113 Sands WA, McNeal JR, Stone MH, Kimmel WL, Haff GG, Jemni M. The effect of vibration on active and passive range of motion in elite female synchronized swimmers. Eur J Sport Sci 2008; 8: 217-223

114 Savelberg H, Keizer HA, Meijer K. Whole-body vibration induced adaptation in knee extensors; Consequences of initial strengths vibration frequency, and joint angle. J Strength Cond Res 2007; 21: 589-593

115 Schuhfried O, Mittermaier C, Jovanovic T, Pieber K, Paternostro-Sluga $T$. Effects of whole-body vibration in patients with multiple sclerosis: a pilot study. Clin Rehabil 2005; 19: 834-842

116 Schyns F, Paul L, Finlay K, Ferguson C, Noble E. Vibration therapy in multiple sclerosis: a pilot study exploring its effects on tone, muscle force, sensation and functional performance. Clin Rehabil 2009; 23: 771-781

117 Segal KR, Edano A, Abalos A, Albu J, Blando L, Tomas MB, Pi-Sunyer $F X$. Effect of exercise training on insulin sensitivity and glucose metabolism in lean, obese, and diabetic men. J Appl Physiol 1991; 71: $2402-2411$

118 Seroussi RE, Wilder DG, Pope $M H$. Trunk muscle electromyography and whole-body vibration. J Biomech 1989; 22: 219-229

119 Silva HR, Couto BP, Szmuchrowski LA. Effects of mechanical vibration applied in the opposite direction of muscle shortening on maximal isometric strength. J Strength Cond Res 2008; 22: 1031-1036

120 Stewart JA, Cochrane DJ, Morton RH. Differential effects of whole body vibration durations on knee extensor strength. J Sci Med Sport 2009; 12: $50-53$

121 Thompson C, Belanger $M$. Effects of vibration in inline skating on the Hoffmann reflex, force, and proprioception. Med Sci Sports Exerc 2002; 34: 2037-2044

122 Tihanyi TK, Horvath M, Fazekas G, Hortobagyi T, Tihanyi J. One session of whole body vibration increases voluntary muscle strength transiently in patients with stroke. Clin Rehabil 2007; 21: 782-793

123 Torvinen S, Kannus P, Sievanen H, Jarvinen TAH, Pasanen M, Kontulainen S, Jarvinen TLN, Jarvinen M, Oja P, Vuori I. Effect of a vibration exposure on muscular performance and body balance. Randomized cross-over study. Clin Physiol Funct Imaging 2002; 22: 145-152
124 Torvinen S, Kannus P, Sievanen H, Jarvinen TAH, Pasanen M, Kontulainen S, Jarvinen TLN, Jarvinen M, Oja P, Vuori I. Effect of four-month vertical whole body vibration on performance and balance. Med Sci Sports Exerc 2002; 34: 1523-1528

125 Torvinen S, Kannus P, Sievanen H, Jarvinen TAH, Pasanen M, Kontulainen S, Nenonen A, Jarvinen TLN, Paakkala T, Jarvinen M, Vuori I. Effect of 8-month vertical whole body vibration on bone, muscle performance, and body balance: A randomized controlled study. J Bone Miner Res 2003; 18: 876-884

126 Torvinen S, Sievanen H, Javinen TAH, Pasanen M, Kontulainen S, Kannus $P$. Effect of 4-min vertical whole body vibration on muscle performance and body balance: a randomized cross-over study. Int J Sports Med 2002; 23: 374-379

127 Trans T, Aaboe J, Henriksen M, Christensen R, Bliddal H, Lund H. Effect of whole body vibration exercise on muscle strength and proprioception in females with knee osteoarthritis. Knee 2009; 16: 256-261

128 Turbanski S, Haas CT, Schmidtbleicher D, Friedrich A, Duisberg P. Effects of random whole-body vibration on postural control in Parkinson's disease. Res Sports Med 2005; 13: 243-256

129 van Nes IJW, Latour H, Schils F, Meijer R, van Kuijk A, Geurts ACH. Long-term effects of 6-week whole-body vibration on balance recovery and activities of daily living in the postacute phase of stroke - A randomized, controlled trial. Stroke 2006; 37: 2331-2335

130 Verschueren SMP, Roelants M, Delecluse C, Swinnen S, Vanderschueren $D$, Boonen S. Effect of 6-month whole body vibration training on hip density, muscle strength, and postural control in postmenopausal women: A randomized controlled pilot study. J Bone Miner Res 2004; 19: 352-359

131 Wakeling JM, Nigg BM, Rozitis AI. Muscle activity damps the soft tissue resonance that occurs in response to pulsed and continuous vibrations. J Appl Physiol 2002; 93: 1093-1103

132 Warman G, Humphries B, Purton J. The effects of timing and application of vibration on muscular contractions. Aviat Space Environ Med 2002; 73: $119-127$

133 Wilcock IM, Whatman C, Harris N, Keogh JWL. Vibration training: Could it enhance the strength, power, or speed of athletes? J Strength Cond Res 2009; 23: 593-603

134 Yue Z, Mester J. A model analysis of internal loads, energetics, and effects of wobbling mass during the whole-body vibration. J Biomech 2002; 35: 639-647 